\title{
Palais-Smale sequences for the fractional CR Yamabe functional and multiplicity results
}

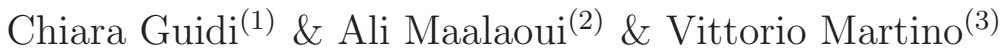

\begin{abstract}
In this paper we consider the functional whose critical points are solutions of the fractional CR Yamabe type equation on the sphere. We firstly study the behaviour of the PalaisSmale sequences characterizing the bubbling phenomena and therefore we prove a multiplicity type result by showing the existence of infinitely many solutions to the related equation.
\end{abstract}

Keywords: fractional sub-elliptic operators, critical exponent.

2010 MSC. Primary: 35J20, 35R11. Secondary: 53A30, 35B33.

\section{Introduction and statement of the results}

Let $N \geq 1$ and let $S^{2 N+1}$ denote the $(2 \mathrm{~N}+1)$-dimensional sphere, equipped with its standard CR structure. In this paper we consider the following energy functional

$$
E(u)=\frac{1}{2} \int_{S^{2 N+1}} u \mathcal{A}_{2 k} u \mathrm{~d} v_{S}-\frac{1}{p^{*}} \int_{S^{2 N+1}}|u|^{p^{*}} \mathrm{~d} v_{S}, \quad u \in H^{k}\left(S^{2 N+1}\right)
$$

whose critical points satisfy the fractional CR Yamabe type equation

$$
\mathcal{A}_{2 k} u=|u|^{p^{*}-2} u \quad \text { on } S^{2 N+1}, \quad u \in H^{k}\left(S^{2 N+1}\right) .
$$

Here $k \in \mathbb{R}$ is a parameter such that $0<2 k<Q:=2 N+2, \mathcal{A}_{2 k}$ is the sub-elliptic intertwining operator of order $2 k$ and $H^{k}\left(S^{2 N+1}\right)$ is the related fractional Sobolev space, as defined for instance in 7, 8, (we will give all the rigorous definitions in Section 2); also, the exponent $p^{*}$ is the critical one for the embedding $H^{k}\left(S^{2 N+1}\right) \hookrightarrow L^{p^{*}}\left(S^{2 N+1}\right)$. Just to fix the ideas, for instance when $k=1$, the operator $\mathcal{A}_{2}$ is nothing but the standard conformal sub-Laplacian on the sphere. Let us also notice that a similar

\footnotetext{
${ }^{1}$ Dipartimento di Matematica, Università di Bologna, piazza di Porta S.Donato 5, 40126 Bologna, Italy. E-mail address: chiara.guidi12@unibo.it

${ }^{2}$ Department of mathematics and natural sciences, American University of Ras Al Khaimah, PO Box 10021, Ras Al Khaimah, UAE. E-mail address: ali.maalaoui@aurak.ae

${ }^{3}$ Dipartimento di Matematica, Università di Bologna, piazza di Porta S.Donato 5, 40126 Bologna, Italy. E-mail address: vittorio.martino3@unibo.it
} 
functional $E_{\mathbb{H}}$ can be defined equivalently on the Heisenberg group $\mathbb{H}^{N}$, via the Cayley transform $\mathcal{C}$, and the related equation on $\mathbb{H}^{N}$ is given by

$$
\mathcal{L}_{2 k} U=|U|^{p^{*}-2} U \quad \text { on } \mathbb{H}^{N}, \quad U \in D^{k}\left(\mathbb{H}^{N}\right) .
$$

We refer the reader to the next section for the definition of $D^{k}\left(\mathbb{H}^{N}\right)$ and the relation between $\mathcal{A}_{2 k}$ and $\mathcal{L}_{2 k}$.

These kind of conformally invariant operators were introduced in [10] and they can be seen as the CR counterpart to the GJMS operators defined in the Riemannian setting in [11]. Indeed, as in the Euclidean case, the existence of an infinite family of explicit positive solutions (bubbles) to the previous equations is known, moreover due to the lack of compactness of the Sobolev embedding (which can be seen geometrically as the action of the conformal group), the functional $E$ does not satisfy the Palais-Smale condition.

However, a sharp Sobolev inequality has been proved by Frank and Lieb in [9], showing that the extremals are exactly the bubbles. All these facts suggest that a characterization of the Palais-Smale sequences should be possible, making the bubbling phenomena completely explicit, as in the classic case.

Indeed, this is what we will prove in our first result. The proof is quite involved and delicate if compared to the standard case: this is due basically to the non-Euclidean setting, the degeneracy of the given operators and also the fractional nature of the problem, making it non-local. As it is commonly known in the standard setting, the bubbling phenomena occurs at a local scale which makes it harder to deal with in a non-local setting. In fact, even if a natural behavior is expected, any variational problem needs a careful analysis depending on the ambient manifold and the structure of the operators involved (see for instance [12, 17]). In our particular case, in addition to the results in 9], we will make use of some point-wise commutator estimates, which has been recently written specifically for these type of operators (see [16]): as is the case of local operators, these kind of estimates are useful in order to study regularity properties, after localizing with cut-off functions (see for instance [4, 22, 14]). Therefore, we will prove the following

Theorem 1.1. Let $u_{n}$ be a Palais-Smale sequence for the functional $E$ at level $c$. Then there exist $u_{\infty}$ a solution of (2), $m$ sequences of points $\zeta_{n}^{1}, \ldots, \zeta_{n}^{m} \in S^{2 N+1}$ such that $\lim _{n \rightarrow \infty} \zeta_{n}^{l}=\zeta^{l} \in S^{2 N+1}$ for $l=1, \ldots, m$ and $m$ sequences of real numbers $R_{n}^{1}, \ldots, R_{n}^{m}$ converging to zero, such that:

$$
\begin{aligned}
& \text { i) } u_{n}=u_{\infty}+\sum_{l=1}^{m} v_{n}^{l}+o(1) \text { in } H^{k}\left(S^{2 N+1}\right) \\
& \text { ii) } E\left(u_{n}\right)=E\left(u_{\infty}\right)+\sum_{l=1}^{m} E_{\mathbb{H}^{N}}\left(U_{\infty}^{l}\right)+o(1)
\end{aligned}
$$

where

$$
v_{n}^{l}=\left(\Lambda_{\sigma_{n}^{l}}\right)^{\frac{Q-2 k}{2 Q}} \beta^{l} U_{\infty}^{l} \circ \sigma_{n}^{l}
$$

with $\sigma_{n}^{l}=\left(\rho_{n}^{l}\right)^{-1}, \rho_{n}^{l}=\mathcal{C} \circ \delta_{R_{n}^{l}} \circ \tau_{\xi_{n}^{l}}$ and $\Lambda_{\sigma_{n}^{l}}$ denote half the absolute value of the Jacobian determinant of $\sigma_{n}^{l} ; \beta^{l}$ are smooth compactly supported functions, such that $\beta^{l} \equiv 1$ on $B_{\frac{1}{4}}\left(\zeta^{l}\right), \operatorname{supp}\left(\beta^{l}\right) \subset B_{1}\left(\zeta^{l}\right)$ and $U_{\infty}^{l}$ are solutions of (3). 
Here the $B$ 's stand for the balls on the sphere, $\delta$ and $\tau$ denote dilations and translations on the Heisenberg group, respectively. The proof of the previous theorem will be carried out in Section 3.

We want explicitly to mention that we recently found a paper on arXiv ([15]), in which the authors prove an existence result for the fractional Q-curvature problem on the three dimensional CR sphere: in their Lemma 2.1, they claim a behavior for PalaisSmale sequences along some flow lines, similar to our Theorem 1.1; the proof is missing, the authors cite a couple of papers, which in turn consider only local operators. To the best of our knowledge, we did not find any references dealing with these peculiar issues that we are considering in the present paper.

Once we have characterized the Palais-Smale sequences, in Section 4 as main application we will prove a multiplicity result for equation (2). We will argue by contradiction as in [19]; in particular, with the help of some special groups of isometries, we will restrict the functional $E$ to some special subspaces and we will assume that the Palais-Smale condition fails: the action of the groups and the boundedness of the energy will lead to a contradiction. Therefore, a standard application of the minimax argument will give us the following result

Theorem 1.2. There exist infinitely many solutions of (3) (or equivalently of (2)), distinct from the standard bubbles.

Moreover, depending on the choice of the group of isometries, the existence of sign changing solutions can be shown. In this setting, we recall the paper [18], where the existence of infinitely many sign changing solutions was proven for the standard CR-Yamabe equation on the sphere (here $k=1$ ), by following the idea of Ding [ $[$ ] combined with the action of the group of isometries generated by the Reeb vector field of the standard sphere. Moreover, recently in [13], under a technical assumption on the range of the parameter $k$, the author proved the existence of a number of sequences of sign-changing solutions of equation (2), whose elements have mutually different nodal properties. The proof is based again on Ding's approach and on a iterative argument as in [2], starting from the result in [18] (the assumption on $k$ makes the iteration works fine). Also, in his Remark 3.2, the author wonders if his technical assumption could be removed in order to gain the compactness of some Sobolev embeddings: it seems that we can remove this assumption and still obtain existence of solutions.

Acknowledgement The second author aknowledge the financial support of the Seed Grant of AURAK, No.: AAS/001/18, Critical Problems in the Sub-Elliptic Setting.

\section{Definitions and notation}

We identify the Heisenberg group $\mathbb{H}^{N}$ with $\mathbb{C}^{N} \times \mathbb{R} \simeq \mathbb{R}^{2 N+1}$ with elements $w=(z, t)=$ $(x+i y, t) \simeq(x, y, t) \in \mathbb{R}^{N} \times \mathbb{R}^{N} \times \mathbb{R}$ and group law

$$
w \cdot w^{\prime}=(z, t) \cdot\left(z^{\prime}, t^{\prime}\right)=\left(z+z^{\prime}, t+t^{\prime}+2 \operatorname{Im}\left(z \overline{z^{\prime}}\right)\right) \quad \forall w, w^{\prime} \in \mathbb{H}^{N},
$$


where Im denotes the imaginary part of a complex number and $z \overline{z^{\prime}}$ is the standard Hermitian inner product in $\mathbb{C}^{N}$. Left translations on $\mathbb{H}^{N}$ are defined by

$$
\tau: \mathbb{H}^{N} \rightarrow \mathbb{H}^{N} \quad \tau_{w}\left(w^{\prime}\right)=w \cdot w^{\prime} \quad \forall w \in \mathbb{H}^{N}
$$

and dilations are

$$
\delta_{\lambda}: \mathbb{H}^{N} \rightarrow \mathbb{H}^{N} \quad \delta_{\lambda}(z, t)=\left(\lambda z, \lambda^{2} t\right) \quad \forall \lambda>0 .
$$

The homogeneous dimension of $\mathbb{H}^{N}$ with respect to $\delta_{\lambda}$ will be denoted by $Q=2 N+2$. The natural distance that we will adopt in our setting is the Korányi distance, given by

$$
d\left((z, t),\left(z^{\prime}, t^{\prime}\right)\right)=\left(\left|z-z^{\prime}\right|^{4}+\left(t-t^{\prime}-2 \operatorname{Im}\left(z \overline{z^{\prime}}\right)\right)^{2}\right)^{\frac{1}{4}}
$$

and we denote by $B_{R}^{w}$ the ball of center $w$ and radius $R>0$ defined by the distance $d$. Moreover we denote by

$$
\theta_{\mathbb{H}}=\mathrm{d} t+2 \sum_{j=1}^{N}\left(x_{i} \mathrm{~d} y_{i}-y_{i} \mathrm{~d} x_{i}\right)
$$

the standard contact form on $\mathbb{H}^{N}$ and by $\mathrm{d} v_{H}$ the volume form associated to $\theta_{\mathbb{H}}$. The canonical basis of left invariant vector fields on $\mathbb{H}^{N}$ is given by

$$
X_{j}=\frac{\partial}{\partial x_{j}}+2 y_{j} \frac{\partial}{\partial t}, \quad Y_{j}=\frac{\partial}{\partial y_{j}}-2 x_{j} \frac{\partial}{\partial t}, \quad T=\frac{\partial}{\partial t}, \quad j=1, \ldots, N .
$$

and the sub-Laplacian operator associated to this Carnot structure is given by

$$
\Delta_{b}=\frac{1}{4} \sum_{j=1}^{N}\left(X_{j}^{2}+Y_{j}^{2}\right)
$$

The Heisenberg group can be identified with the unit sphere in $\mathbb{C}^{N+1}$ minus a point through the Cayley transform $\mathcal{C}: \mathbb{H}^{N} \rightarrow S^{2 N+1} \backslash\{(0, \ldots, 0,-1)\}$ defined as follows

$$
\mathcal{C}(z, t)=\left(\frac{2 z}{1+|z|^{2}+i t}, \frac{1-|z|^{2}-i t}{1+|z|^{2}+i t}\right) \text {. }
$$

On the unit sphere $S^{2 N+1}=\left\{\zeta \in \mathbb{C}^{N+1}:|\zeta|=1\right\}$ we consider the distance

$$
d(\zeta, \eta)^{2}=2|1-\zeta \bar{\eta}|, \quad \zeta, \eta \in \mathbb{C}^{N+1}
$$

and we denote by $B_{R}(\zeta) \subset S^{2 N+1}$ the ball of center $\zeta$ and radius $R>0$. With this definition of $d$, the relation between the distance of two points $w=(z, t), w^{\prime}=\left(z^{\prime}, t^{\prime}\right)$ in $\mathbb{H}^{N}$ and the distance of their images $\mathcal{C}(w), \mathcal{C}\left(w^{\prime}\right)$ in $S^{2 N+1}$, is given by

$$
d\left(\mathcal{C}(w), \mathcal{C}\left(w^{\prime}\right)\right)=d\left(w, w^{\prime}\right)\left(\frac{4}{\left(1+|z|^{2}\right)^{2}+t^{2}}\right)^{\frac{1}{4}}\left(\frac{4}{\left(1+\left|z^{\prime}\right|^{2}\right)^{2}+t^{\prime 2}}\right)^{\frac{1}{4}} .
$$


From this relation we deduce the following inclusions

$$
\mathcal{C}^{-1}\left(B_{R}(\zeta)\right) \supseteq B_{\frac{R}{2}}^{\mathcal{C}^{-1}(\zeta)} \quad \text { for every } R>0
$$

and

$$
\mathcal{C}^{-1}\left(B_{R}(\mathcal{N})\right) \subseteq B_{R}^{0} \quad \text { for every } 1 \geq R>0
$$

where $\mathcal{N}$ is the point $(1,0, \ldots, 0) \in S^{2 N+1}$. On $S^{2 N+1}$, we consider the standard contact form

$$
\theta_{S}=i \sum_{j=1}^{N+1}\left(\zeta_{j} \mathrm{~d} \bar{\zeta}_{j}-\bar{\zeta}_{j} \mathrm{~d} \zeta_{j}\right)
$$

and we denote by $\mathrm{d} v_{S}$ the volume form associated to $\theta_{S}$. The conformal sub-Laplacian is then

$$
\mathcal{A}_{2}=-\frac{1}{2} \sum_{j=1}^{N+1}\left(T_{j} \bar{T}_{j}+\bar{T}_{j} T_{j}\right)+\frac{N^{2}}{4}
$$

where $T_{j}$ are the differential operators defined by

$$
T_{j}=\frac{\partial}{\partial \zeta_{j}}-\overline{\zeta_{j}} \sum_{k=1}^{N+1} \zeta_{k} \frac{\partial}{\partial \zeta_{k}}, j=1, \ldots, N+1 .
$$

Let $\mathcal{H}_{j, l}$ be the space of harmonic polynomials on $\mathbb{C}^{N+1}$ homogeneous of degree $j$ and $l$ in variables $z$ and $\bar{z}$ respectively, restricted to $S^{2 N+1}$. The Hilbert space $L^{2}\left(S^{2 N+1}\right)$ decomposes as $L^{2}\left(S^{2 N+1}\right)=\bigoplus_{j, l \geq 0} \mathcal{H}_{j, l}$ and we denote by $y_{j, l}^{m}$ an orthonormal basis for the space $\mathcal{H}_{j, l}$, in particular we require $y_{j, l}^{m}$ to be eigenfunction for the conformal sub-Laplacian $\mathcal{A}_{2}$. Then, the conformal sub-Laplacian acts on $y_{j, l}^{m}$ as $\mathcal{A}_{2} y_{j, l}^{m}=\lambda_{j} \lambda_{l} y_{j, l}^{m}$, where $\lambda_{j}=j+\frac{n}{2}$. Let us fix $0<2 k<Q$, and consider

$$
u=\sum_{j, l} \sum_{m=1}^{\operatorname{dim}\left(\mathcal{H}_{j, l}\right)} c_{j, l}^{m}(u) y_{j, l}^{m} \in L^{2}\left(S^{2 N+1}\right) .
$$

We define the operator

$$
\mathcal{A}^{k} u=\sum_{j, l} \sum_{m=1}^{\operatorname{dim}\left(\mathcal{H}_{j, l}\right)}\left(\lambda_{j} \lambda_{l}\right)^{\frac{k}{2}} c_{j, l}^{m}(u) y_{j, l}^{m}
$$

where $\operatorname{dim}\left(\mathcal{H}_{j, l}\right)=\frac{(j+N-1) !(l+N-1) !(j+l+N)}{N !(N-1) ! j ! ! !}$ is the dimension of $\mathcal{H}_{j, l}$. Moreover, we define the Sobolev space

$$
H^{k}\left(S^{2 N+1}\right)=\left\{u \in L^{2}\left(S^{2 N+1}\right): \mathcal{A}^{k} u \in L^{2}\left(S^{2 N+1}\right)\right\}
$$

with inner product

$$
\langle u, v\rangle_{k}=\int_{S^{2 N+1}} \mathcal{A}^{k} u \overline{\mathcal{A}^{k} v} \mathrm{~d} v_{S}
$$


and norm

$$
\|u\|_{k}=\langle u, u\rangle_{k}^{\frac{1}{2}}=\left(\sum_{j, l} \sum_{m=1}^{\operatorname{dim}\left(\mathcal{H}_{j, l}\right)}\left(\lambda_{j} \lambda_{l}\right)^{k}\left|c_{j, l}^{m}(u)\right|^{2}\right)^{\frac{1}{2}}
$$

We consider the intertwining operator $\mathcal{A}_{2 k}$ on $S^{2 N+1}$ defined, up to multiplicative constants, by

$$
\operatorname{Jac}_{\tau}^{\frac{Q+2 k}{2 Q}}\left(\mathcal{A}_{2 k} u\right) \circ \tau=\mathcal{A}_{2 k}\left(\operatorname{Jac}_{\tau}^{\frac{Q-2 k}{2 Q}}(u \circ \tau)\right) \quad \forall \tau \in \operatorname{Aut}\left(S^{2 N+1}\right), u \in C^{\infty}\left(S^{2 N+1}\right) .
$$

Moreover from now on we endow $H^{k}\left(S^{2 N+1}\right)$ with the inner product

$$
\langle u, v\rangle_{H^{k}}=\sum_{j, l} \sum_{m=1}^{\operatorname{dim}\left(\mathcal{H}_{j}\right)}\left(\lambda_{j}(k)\right)^{2 k} c_{j}^{m}(u) \overline{c_{j}^{m}(v)}=\int_{S^{2 N+1}} \bar{v} \mathcal{A}_{2 k} u \mathrm{~d} v_{S}
$$

with

$$
\lambda_{j}(k)=\frac{\Gamma\left(\frac{Q+2 k}{4}+j\right)}{\Gamma\left(\frac{Q-2 k}{4}+j\right)}, \quad j=0,1, \ldots
$$

and norm $\|u\|_{H^{k}}=\left(\int_{S^{2 N+1}} \bar{u} \mathcal{A}_{2 k} u \mathrm{~d} v_{S}\right)^{\frac{1}{2}}$ which is equivalent to $\|u\|_{k}$. The dual of $H^{k}\left(S^{2 N+1}\right)$ will be denoted by $H^{-k}$. In $\mathbb{H}^{N}$ the symbol of the intertwining operators is defined, up to a multiplicative constant, by

$$
\mathcal{L}_{2 k}=|2 T|^{k} \frac{\Gamma\left(\frac{-\Delta_{b}}{|2 T|}+\frac{1+k}{2}\right)}{\Gamma\left(\frac{-\Delta_{b}}{|2 T|}+\frac{1-k}{2}\right)} ;
$$

we choose the multiplicative constant to be equal 1 so that we recover $\mathcal{L}_{2}=-\Delta_{b}$ and $\mathcal{L}_{4}=\left(-\Delta_{b}\right)^{2}-T^{2}$. Hereafter we consider only real valued functions. The quadratic form associated to $\mathcal{L}_{2 k}$ will be denoted by $a_{2 k}$ :

$$
a_{2 k}(U)=\int_{\mathbb{H}^{N}} U \mathcal{L}_{2 k} U \mathrm{~d} v_{H}
$$

and we define the space

$$
D^{k}\left(\mathbb{H}^{N}\right)=\left\{U \in L^{\frac{2 Q}{Q-2 k}}\left(\mathbb{H}^{N}\right): a_{2 k}<+\infty\right\} .
$$

The operators $\mathcal{A}_{2 k}$ and $\mathcal{L}_{2 k}$ are related by the following identity

$$
\mathcal{L}_{2 k}\left(\Lambda_{\mathcal{C}}^{\frac{Q-2 k}{2 Q}}(u \circ \mathcal{C})\right)=\Lambda_{\mathcal{C}}^{\frac{Q+2 k}{2 Q}}\left(\mathcal{A}_{2 k} u\right) \circ \mathcal{C} \quad \forall u \in H^{k}\left(S^{2 N+1}\right)
$$

where $\Lambda_{\mathcal{C}}$ is twice the absolute value of the Jacobian determinant of the Cayley transform

$$
\Lambda_{\mathcal{C}}=\frac{2^{Q}}{\left(\left(1+|z|^{2}\right)^{2}+t^{2}\right)^{N+1}} .
$$


We recall now the following sharp Sobolev inequality that was proved by Frank and Lieb in 9 ]

$$
\left(\int_{S^{2 N+1}}|u|^{\frac{2 Q}{Q-2 k}} \mathrm{~d} v_{S}\right)^{\frac{Q-2 k}{Q}} \leq C_{S} \int_{S^{2 N+1}} u \mathcal{A}_{2 k} u \mathrm{~d} v_{S}
$$

where

$$
C_{S}(k, N)=\frac{\Gamma\left(\frac{N+1-k}{2}\right)^{2}}{\Gamma\left(\frac{N+1+k}{2}\right)^{2}}\left(\omega_{2 N+1} 2^{2 N+1} N !\right)^{-\frac{2 k}{Q}},
$$

$\omega_{2 N+1}$ is the measure of $S^{2 N+1}$ and

$$
p^{*}=\frac{2 Q}{Q-2 k}
$$

is the critical exponent. Indeed the embedding

$$
H^{k}\left(S^{2 N+1}\right) \hookrightarrow L^{p^{*}}\left(S^{2 N+1}\right)
$$

is continuous but not compact and this is due to the scale invariance of the norms, induced by the action of the conformal group. Also, we will denote by $\bar{p}=\left(p^{*}\right)^{\prime}=\frac{2 Q}{Q+2 k}$ and it follows from (9) that

$$
L^{\bar{p}}\left(S^{2 N+1}\right) \hookrightarrow H^{-k}\left(S^{2 N+1}\right) .
$$

For $\Omega \subset \mathbb{H}^{N}$ open and bounded we denote by $H_{0}^{k}(\Omega)$ the closure of $C_{0}^{\infty}(\Omega)$ with respect to the norm

$$
\|U\|_{H_{0}^{k}(\Omega)}=\left(\int_{\Omega} U \mathcal{L}_{2 k} U \mathrm{~d} v_{H}\right)^{\frac{1}{2}}
$$

and it holds

$$
H_{0}^{k}(\Omega) \hookrightarrow L^{p^{*}}(\Omega)
$$

Optimizer functions for (77) are images through the Cayley transform of functions of the type $\lambda^{\frac{2 k-Q}{2}} \omega \circ \delta_{\lambda^{-1}} \circ \tau_{\xi^{-1}}$ where

$$
\omega(z, t)=\frac{c(Q)}{\left(\left(1+|z|^{2}\right)^{2}+t^{2}\right)^{\frac{Q-2 k}{4}}},
$$

for a suitable positive constant $c(Q)$ (see [9]). These functions satisfy the equation

$$
\mathcal{L}_{2 k} U=|U|^{p^{*}-2} U \quad \text { on } \mathbb{H}^{N} \quad U \in D^{k}\left(\mathbb{H}^{N}\right),
$$

hence they are critical points for the energy functional $E_{\mathbb{H}}$ defined on $D^{k}\left(\mathbb{H}^{N}\right)$ by

$$
E_{\mathbb{H}}(U)=\frac{1}{2} \int_{\mathbb{H}^{N}} U \mathcal{L}_{2 k} U \mathrm{~d} v_{H}-\frac{1}{p^{*}} \int_{\mathbb{H}^{N}}|U|^{p^{*}} \mathrm{~d} v_{H}
$$

In fact the functions $\omega_{\lambda, \xi}=\lambda^{\frac{2 k-Q}{2}} \omega \circ \delta_{\lambda^{-1}} \circ \tau_{\xi^{-1}}$ are (the only) ground state solutions of $E_{\mathbb{H}}$. 


\section{Classification of the Palais-Smale sequences}

Let $H$ be an Hilbert space, a sequence $\left\{x_{n}\right\}_{n \in \mathbb{N}} \subseteq H$ is called a Palais-Smale (PS) sequence for $F \in C^{1}(H, \mathbb{R})$ at level $c$ if $F\left(x_{n}\right) \rightarrow c$ and $\nabla F\left(x_{n}\right) \rightarrow 0$. $F$ is said to satisfy the Palais-Smale condition if any (PS) sequence admits a converging subsequence.

Now, we begin the proof of our main result, that is Theorem 1.1.

Lemma 3.1. Every (PS) sequence $u_{n}$ for $E$ is bounded.

Proof. Let $u_{n}$ be a (PS) sequence for $E$ at level $c$ i.e.

$$
E\left(u_{n}\right) \rightarrow c, \quad d E\left(u_{n}\right) \rightarrow 0 \text { in } H^{-k}\left(S^{2 N+1}\right) .
$$

Therefore we have

$$
\begin{aligned}
2 c+o(1)+o(1)\left\|u_{n}\right\|_{H^{k}} & \geq 2 E\left(u_{n}\right)-\left\langle d E\left(u_{n}\right), u_{n}\right\rangle \\
& =\left(\frac{p^{*}-2}{p^{*}}\right) \int_{S^{2 N+1}}\left|u_{n}\right|^{p^{*}} \mathrm{~d} v_{S},
\end{aligned}
$$

hence

$$
\begin{aligned}
\left\|u_{n}\right\|_{H^{k}}^{2} & =2 E\left(u_{n}\right)+\frac{2}{p^{*}} \int_{S^{2 N+1}}\left|u_{n}\right|^{p^{*}} \mathrm{~d} v_{S} \\
& \leq 2 c+o(1)+\frac{2}{p^{*}-2}\left(2 c+o(1)+o(1)\left\|u_{n}\right\|_{H^{k}}\right) .
\end{aligned}
$$

It follows that $u_{n}$ is bounded in $H^{k}\left(S^{2 N+1}\right)$.

The result above implies that, up to a subsequence, there exists a function $u_{\infty} \in$ $H^{k}\left(S^{2 N+1}\right)$ such that

$$
\begin{array}{ll}
u_{n} \rightarrow u_{\infty} & \text { weakly in } H^{k}\left(S^{2 N+1}\right), \\
u_{n} \rightarrow u_{\infty} & \text { strongly in } L^{p}\left(S^{2 N+1}\right) \quad \text { for } 1 \leq p<p^{*} .
\end{array}
$$

Moreover, $u_{\infty}$ is a weak solution to (2). Indeed, since $u_{n}$ is a (PS) sequence for $E$, for any $\varphi \in H^{k}\left(S^{2 N+1}\right)$ we have

$$
\int_{S^{2 N+1}} \varphi \mathcal{A}_{2 k} u_{n} \mathrm{~d} v_{S}=\int_{S^{2 N+1}} \varphi\left|u_{n}\right|^{p^{*}-2} u_{n} \mathrm{~d} v_{S}+o(1)
$$

as $n \rightarrow \infty$, and by (12) and (11) we have respectively

$$
\begin{aligned}
\int_{S^{2 N+1}} \varphi \mathcal{A}_{2 k} u_{n} \mathrm{~d} v_{S} & \rightarrow \int_{S^{2 N+1}} \varphi \mathcal{A}_{2 k} u_{\infty} \mathrm{d} v_{S} \\
\int_{S^{2 N+1}} \varphi\left|u_{n}\right|^{p^{*}-2} u_{n} \mathrm{~d} v_{S} & \rightarrow \int_{S^{2 N+1}} \varphi\left|u_{\infty}\right|^{p^{*}-2} u_{\infty} \mathrm{d} v_{S}
\end{aligned}
$$

showing that $u_{\infty}$ weakly satisfies (2). We set $v_{n}=u_{n}-u_{\infty}$, with this notation we have the following 
Lemma 3.2. The sequence $v_{n}$ is a (PS) sequence for E. More precisely, it holds

$$
E\left(v_{n}\right)=E\left(u_{n}\right)-E\left(u_{\infty}\right)+o(1)
$$

and

$$
d E\left(v_{n}\right) \rightarrow 0, \quad \text { in } H^{-k}\left(S^{2 N+1}\right)
$$

Proof. We have

$$
\begin{aligned}
2 E\left(u_{n}\right)= & \int_{S^{2 N+1}}\left(v_{n}+u_{\infty}\right) \mathcal{A}_{2 k}\left(v_{n}+u_{\infty}\right) \mathrm{d} v_{S}-\frac{2}{p^{*}} \int_{S^{2 N+1}}\left|v_{n}+u_{\infty}\right|^{p^{*}} \mathrm{~d} v_{S} \\
= & 2 E\left(v_{n}\right)+2 E\left(u_{\infty}\right)+2\left\langle d E\left(u_{\infty}\right), v_{n}\right\rangle+2 \int_{S^{2 N+1}}\left|u_{\infty}\right|^{p^{*}-2} u_{\infty} v_{n} \mathrm{~d} v_{S}+ \\
& \quad+\frac{2}{p^{*}} \int_{S^{2 N+1}}\left|v_{n}\right|^{p^{*}}+\left|u_{\infty}\right|^{p^{*}}-\left|v_{n}+u_{\infty}\right|^{p^{*}} \mathrm{~d} v_{S} .
\end{aligned}
$$

Since $v_{n} \rightarrow 0$ in $L^{p}$ for every $1 \leq p<p^{*}$, we have $\int_{S^{2 N+1}}\left|u_{\infty}\right|^{p^{*}-2} u_{\infty} v_{n} \mathrm{~d} v_{S}=o(1)$ as $n \rightarrow \infty$; moreover $d E\left(u_{\infty}\right)=0$, so that it remains to show that the last integral in the expression above goes to 0 as $n \rightarrow \infty$. It is possible to choose a big enough positive constant $C$ such that

$$
\left|\Phi_{n}\right|:=|| v_{n}+\left.u_{\infty}\right|^{p^{*}}-\left|v_{n}\right|^{p^{*}}-\left.\left|u_{\infty}\right|^{p^{*}}|\leq C| v_{n}\right|^{p^{*}-1}\left|u_{\infty}\right|+C\left|v_{n}\right|\left|u_{\infty}\right|^{p^{*}-1} .
$$

Hence, by the Hölder inequality

$$
\begin{aligned}
\int_{S^{2 N+1}}\left|\Phi_{n}\right| \mathrm{d} v_{S}= & \int_{S^{2 N+1} \backslash M_{\epsilon}}\left|\Phi_{n}\right| \mathrm{d} v_{S}+\int_{M_{\epsilon}}\left|\Phi_{n}\right| \mathrm{d} v_{S} \\
\leq & \int_{S^{2 N+1} \backslash M_{\epsilon}}\left|\Phi_{n}\right| \mathrm{d} v_{S}+C\left(\int_{M_{\epsilon}}\left|v_{n}\right|^{p^{*}} \mathrm{~d} v_{S}\right)^{\frac{p^{*}-1}{p^{*}}}\left(\int_{M_{\epsilon}}\left|u_{\infty}\right|^{p^{*}} \mathrm{~d} v_{S}\right)^{\frac{1}{p^{*}}} \\
& +C\left(\int_{M_{\epsilon}}\left|u_{\infty}\right|^{p^{*}} \mathrm{~d} v_{S}\right)^{\frac{p^{*}-1}{p^{*}}}\left(\int_{M_{\epsilon}}\left|v_{n}\right|^{p^{*}} \mathrm{~d} v_{S}\right)^{\frac{1}{p^{*}}} .
\end{aligned}
$$

Here $M_{\epsilon} \subset S^{2 N+1}$, defined for any $\epsilon>0$ by the Egorov theorem, is such that $\mid S^{2 N+1} \backslash$ $M_{\epsilon} \mid<\epsilon$ and $v_{n}$ converges to 0 uniformly on $M_{\epsilon}$. So that, the first integral in the expression above converges to 0 as $n \rightarrow \infty$, while the other two terms go to 0 as $\epsilon \rightarrow 0$, uniformly in $n$. Therefore we get the desired energy estimate. Now we prove that for any $\varphi \in H^{k}\left(S^{2 N+1}\right)$ with $\|\varphi\|_{H^{k}\left(S^{2 N+1}\right)} \leq 1$, it holds

$$
\left\langle d E\left(v_{n}\right), \varphi\right\rangle=o(1) \quad \text { as } n \rightarrow \infty .
$$

We have

$$
\begin{aligned}
\left\langle d E\left(u_{n}\right), \varphi\right\rangle= & \int_{S^{2 N+1}} \varphi \mathcal{A}_{2 k}\left(v_{n}+u_{\infty}\right) \mathrm{d} v_{S}-\int_{S^{2 N+1}}\left|u_{n}\right|^{p^{*}-2} u_{n} \varphi \mathrm{d} v_{S} \\
= & \left\langle d E\left(v_{n}\right), \varphi\right\rangle+\left\langle d E\left(u_{\infty}\right), \varphi\right\rangle+ \\
& \quad-\int_{S^{2 N+1}}\left(\left|u_{n}\right|^{p^{*}-2} u_{n}-\left|v_{n}\right|^{p^{*}-2} v_{n}-\left|u_{\infty}\right|^{p^{*}-2} u_{\infty}\right) \varphi \mathrm{d} v_{S} .
\end{aligned}
$$


Since $\left\langle d E\left(u_{\infty}\right), \varphi\right\rangle=0$ and $\left\langle d E\left(u_{n}\right), \varphi\right\rangle=o(1)$ as $n \rightarrow \infty$, it remains to show that the last integral in the equality above converges to 0 as $n \rightarrow \infty$. Again, for a big enough positive constant $C$ we have

$$
\left|\Psi_{n}\right|:=\left.|| u_{n}\right|^{p^{*}-2} u_{n}-\left|v_{n}\right|^{p^{*}-2} v_{n}-\left.\left|u_{\infty}\right|^{p^{*}-2} u_{\infty}|\leq C| v_{n}\right|^{p^{*}-2}\left|u_{\infty}\right|+C\left|v_{n}\right|\left|u_{\infty}\right|^{p^{*}-2}
$$

and by the Hölder inequality and Egorov theorem

$$
\begin{aligned}
\left|\int_{S^{2 N+1}} \Psi_{n}\right| \varphi\left|\mathrm{d} v_{S}\right| & \lesssim \int_{S^{2 N+1}}\left|v_{n}\right|^{p^{*}-2}\left|u_{\infty}\right||\varphi| \mathrm{d} v_{S}+\int_{S^{2 N+1}}\left|v_{n} \| u_{\infty}\right|^{p^{*}-2} \varphi \mathrm{d} v_{S} \\
& \lesssim\left\|\left|v_{n}\right|^{p^{*}-2}\left|u_{\infty}\right|\right\|_{L^{\frac{p^{*}}{p^{*}-1}}}\|\varphi\|_{L^{p^{*}}}+\left\|\left|v_{n}\left\|\left.u_{\infty}\right|^{p^{*}-2}\right\|_{L^{\frac{p^{*}}{p^{*}-1}}}\|\varphi\|_{L^{p^{*}}}\right.\right. \\
& \lesssim\left\|\left|v_{n}\right|^{p^{*}-2}\left|u_{\infty}\right|\right\|_{L^{\frac{p^{*}}{p^{*}-1}}}+\left\|\left|v_{n}\left\|\left.u_{\infty}\right|^{p^{*}-2}\right\|_{L^{\frac{p^{*}}{p^{*}-1}}}\right.\right. \\
& \lesssim\left\|\left|v_{n}\right|^{p^{*}-2}\left|u_{\infty}\right|\right\|_{L^{\frac{p^{*}}{p^{*}-1}}\left(S^{2 N+1} \backslash M_{\epsilon}\right)}+\left\|\left|v_{n}\left\|\left.u_{\infty}\right|^{p^{*}-2}\right\|_{L^{\frac{p^{*}}{p^{*}-1}}\left(S^{2 N+1} \backslash M_{\epsilon}\right)}\right.\right. \\
& +\left\|\left|v_{n}\right|^{p^{*}-2}\left|u_{\infty}\right|\right\|_{L^{\frac{p^{*}}{p^{*}-1}}\left(M_{\epsilon}\right)}+\left\|\left|v_{n}\left\|\left.u_{\infty}\right|^{p^{*}-2}\right\|_{L^{\frac{p^{*}}{p^{*}-1}}\left(M_{\epsilon}\right)}\right.\right. \\
& =o(1) \text { as } n \rightarrow \infty \text { and } \epsilon \rightarrow 0, \text { uniformly in } n .
\end{aligned}
$$

This concludes the proof.

Lemma 3.3. Let $u_{n}$ be a $(P S)$ sequence at level $c<\frac{k}{Q} C_{S}^{-\frac{Q}{2 k}}$, then $u_{n}$ converges strongly to 0 in $H^{k}\left(S^{2 N+1}\right)$. Here $C_{S}$ is the Sobolev constant defined in (8).

Proof. By the Sobolev inequality (7), we have $\left\|u_{n}\right\|_{L^{p^{*}}}^{p^{*}} \leq C_{S}^{\frac{p^{*}}{2}}\left\|u_{n}\right\|_{H^{k}}^{p^{*}}$, so that

$$
\begin{aligned}
o(1) & =\int_{S^{2 N+1}} u_{n} \mathcal{A}_{2 k} u_{n} \mathrm{~d} v_{S}-\int_{S^{2 N+1}}\left|u_{n}\right|^{p^{*}} \mathrm{~d} v_{S} \\
& \geq\left\|u_{n}\right\|_{H^{k}}^{2}\left(1-C_{S}^{\frac{p^{*}}{2}}\left\|u_{n}\right\|_{H^{k}}^{p^{*}-2}\right) .
\end{aligned}
$$

Now, following the argument given in Lemma 3.1, we notice that the choice of $c$ in the statement implies

$$
\begin{aligned}
\left\|u_{n}\right\|_{H^{k}}^{2} & \leq \frac{p^{*}}{p^{*}-2} 2 c+o(1) \\
& <C_{S}^{-\frac{Q}{2 k}}+o(1) .
\end{aligned}
$$

This, for $n$ big enough, ensures the positivity of the factor $1-C_{S}^{\frac{p^{*}}{2}}\left\|u_{n}\right\|_{H^{k}}^{p^{*}-2}$, concluding the proof.

Hereafter we assume the (PS) sequence $\left(u_{n}\right)_{n \in \mathbb{N}}$ converges weakly to 0 in $H^{k}\left(S^{2 N+1}\right)$ and strongly in $L^{p}\left(S^{2 N+1}\right)$ for $1 \leq p<p^{*}$. Moreover, since we want to investigate the 
behavior of $\left(u_{n}\right)$ when the (PS) condition is not satisfied, we will assume that $u_{n}$ does not converge strongly to 0 in $H^{k}\left(S^{2 N+1}\right)$. For any $\epsilon_{0}>0$, we define

$$
\Sigma_{\epsilon_{0}}=\left\{\zeta \in S^{2 N+1}: \liminf _{r \rightarrow 0} \liminf _{n \rightarrow \infty} \int_{B_{r}(\zeta)}\left|u_{n}\right|^{p^{*}} \mathrm{~d} v_{S} \geq \epsilon_{0}\right\} .
$$

In the sequel we will need to localize our equation, therefore we will use some commutator estimates. For a given $k \in\left(0, \frac{Q}{2}\right)$, we define the 3 -commutator $H^{\mathcal{L}_{2 k}}(\cdot, \cdot)$ by

$$
H^{\mathcal{L}_{2 k}}(u, v)=\mathcal{L}_{2 k}(u v)-u \mathcal{L}_{2 k}(v)-v \mathcal{L}_{2 k}(u)
$$

and we let $R_{s}$ denote the Riesz potential on $\mathbb{H}^{N}$ (see Appendix). Then the following Lemma [16] holds

Lemma (commutator estimates). Let $0<2 k<Q$ and $\epsilon>0$. Given $\tau_{1}$ and $\tau_{2}$ in $(\max \{0,2 k-1\}, 2 k]$ such that $\tau_{1}+\tau_{2}>2 k$, there exists $L \in \mathbb{N}, s_{j, 1} \in\left(0, \tau_{1}\right)$, $s_{j, 2} \in\left(0, \tau_{2}\right)$, for $j=1, \cdots, L$, satisfying $\tau_{1}+\tau_{2}-s_{j, 1}-s_{j, 2}-2 k \in[0, \epsilon)$ such that

$$
\left|H^{\mathcal{L}_{2 k}}(u, v)\right|(x) \lesssim \sum_{j=1}^{L} R_{\tau_{1}+\tau_{2}-s_{j, 1}-s_{j, 2}-2 k}\left(R_{s_{j, 1}}\left|\mathcal{L}_{\tau_{1}} u\right| R_{s_{j, 2}}\left|\mathcal{L}_{\tau_{2}} v\right|\right)(x) .
$$

Next, we state the first Lemma characterizing the concentration set:

Lemma 3.4. There exists $\epsilon_{0}>0$ such that if $\zeta_{0} \notin \Sigma_{\epsilon_{0}}$, then for a small enough $r>0$, we have $u_{n} \rightarrow 0$ in $H^{k}\left(B_{r}\left(\zeta_{0}\right)\right)$.

Proof. Suppose by contradiction that for every $\epsilon>0$ there exists $\zeta_{0} \notin \Sigma_{\epsilon}$ such that $u_{n}$ does not converges to 0 in $H^{k}\left(B_{r}\left(\zeta_{0}\right)\right)$ for every $r>0$. Notice that $\zeta_{0} \notin \Sigma_{\epsilon}$ implies the existence of a radius $r>0$ such that

$$
\int_{B_{2 r}\left(\zeta_{0}\right)}\left|u_{n}\right|^{p^{*}} \mathrm{~d} v_{S}<\epsilon
$$

Since $u_{n}$ is a (PS) sequence, there exists a sequence $\delta_{n} \in H^{-k}\left(S^{2 N+1}\right)$ converging to 0 in $H^{-k}\left(S^{2 N+1}\right)$, such that

$$
\mathcal{A}_{2 k}\left(u_{n}\right)=\left|u_{n}\right|^{p^{*}-2} u_{n}+\delta_{n}
$$

Since we want to localize around $\zeta_{0}$, we consider the Cayley transform $\mathcal{C}$ where we set the north pole as $\zeta_{0}$. We notice that for any couple of functions $u, v$ it holds

$$
\mathcal{A}_{2 k}(u v)=\Lambda_{\mathcal{C}}^{-\frac{1}{\bar{p}}}\left[\mathcal{L}_{2 k}\left(\Lambda_{\mathcal{C}}^{\frac{1}{p^{*}}}(u v) \circ \mathcal{C}\right)\right] \circ \mathcal{C}^{-1}
$$

and

$$
\mathcal{L}_{2 k}\left(\Lambda_{\mathcal{C}}^{\frac{1}{p^{*}}}(u v) \circ \mathcal{C}\right)=v \circ \mathcal{C} \mathcal{L}_{2 k}\left(\Lambda_{\mathcal{C}}^{\frac{1}{p^{*}}} u \circ \mathcal{C}\right)+\Lambda_{\mathcal{C}}^{\frac{1}{p^{*}}} u \circ \mathcal{C} \mathcal{L}_{2 k}(v \circ \mathcal{C})+H^{\mathcal{L}_{2 k}}\left(\Lambda_{\mathcal{C}}^{\frac{1}{p^{*}}} u \circ \mathcal{C}, v \circ \mathcal{C}\right)
$$


We go back now to our Palais-Smale sequence and we compute

$$
\begin{aligned}
\mathcal{A}_{2 k}\left(\eta u_{n}\right) & =\eta \mathcal{A}_{2 k}\left(u_{n}\right)+\text { l.o.t. } \\
& =\eta\left|u_{n}\right|^{p^{*}-2} u_{n}+\eta \delta_{n}+\text { l.o.t. }
\end{aligned}
$$

Here $\eta$ is a smooth cut off function with $\operatorname{supp}(\eta) \subseteq B_{2 r}\left(\zeta_{0}\right)$ and $\eta \equiv 1$ on $B_{r}\left(\zeta_{0}\right)$. So we first estimate $\|$ l.o.t $\|_{H^{-k}}$. We have

$$
\begin{aligned}
\left|H_{k}\left(\Lambda_{\mathcal{C}}^{\frac{1}{p^{*}}} u_{n} \circ \mathcal{C}, \eta \circ \mathcal{C}\right)\right| & \lesssim \sum_{i=1}^{L} R_{2 k-s_{i}-t_{i}}\left(R_{t_{i}}\left(\mathcal{L}_{2 k}\left(\Lambda_{\mathcal{C}}^{\frac{1}{p^{*}}} u_{n} \circ \mathcal{C}\right)\right) R_{s_{i}}\left(\mathcal{L}_{2 k}(\eta \circ \mathcal{C})\right)\right) \\
& \lesssim \sum_{i=1}^{L} R_{2 k-s_{i}-t_{i}}\left(R_{t_{i}}\left(\left(\tilde{u}_{n}+\delta_{n}\right) \circ \mathcal{C}\right) R_{s_{i}}\left(\mathcal{L}_{2 k}(\eta \circ \mathcal{C})\right)\right)
\end{aligned}
$$

where $\tilde{u}_{n}=\left|\Lambda_{\mathcal{C}}^{\frac{1}{p^{*}}} u_{n}\right|^{p^{*}-2} \Lambda_{\mathcal{C}}^{\frac{1}{p^{*}}} u_{n}$. Since the terms of the sumation above are similar in nature we will give here the proof for a single term. Since $\tilde{u}_{n}$ is bounded in $L^{\bar{p}}$, we have that $R_{t}\left(\tilde{u}_{n} \circ \mathcal{C}\right)$ converges strongly to zero (up to a subsequence) in $L_{l o c}^{q}$ for $\frac{1}{q}>\frac{1}{\bar{p}}-\frac{t}{Q}$. So we fix $R>0$ big enough. Then we have

$$
\left\|R_{2 k-s-t}\left(R_{t}\left(\tilde{u}_{n} \circ \mathcal{C}\right) \chi_{B_{R}^{0}} R_{s}\left(\mathcal{L}_{2 k}(\eta \circ \mathcal{C})\right)\right)\right\|_{L^{\bar{p}}} \lesssim\left\|R_{t}\left(\tilde{u}_{n} \circ \mathcal{C}\right)\right\|_{L^{q}\left(B_{R}^{0}\right)}\left\|\mathcal{L}_{2 k}(\eta \circ \mathcal{C})\right\|_{L^{p}}
$$

where $\frac{1}{\bar{p}}=\frac{1}{q}+\frac{1}{p}-\frac{2 k-t}{Q}$. Hence

$$
\left\|R_{2 k-s-t}\left(R_{t}\left(\tilde{u}_{n} \circ \mathcal{C}\right) \chi_{B_{R}^{0}} R_{s}\left(\mathcal{L}_{2 k}(\eta \circ \mathcal{C})\right)\right)\right\|_{L^{\bar{p}}} \rightarrow 0 .
$$

Outside $B_{R}^{0}$, we have that

$$
\chi_{\mathbb{H}^{N} \backslash B_{R}^{0}} R_{s}\left(\mathcal{L}_{2 k}(\eta \circ \mathcal{C})\right)(x) \lesssim \frac{1}{|x|^{Q+2 k-s}}
$$

Thus,

$$
\left\|R_{2 k-s-t}\left(R_{t}\left(\tilde{u}_{n} \circ \mathcal{C}\right) \chi_{\mathbb{H}^{N} \backslash B_{R}^{0}} R_{s}\left(\mathcal{L}_{2 k}(\eta \circ \mathcal{C})\right)\right)\right\|_{L^{\bar{p}}} \lesssim\left\|\tilde{u}_{n} \circ \mathcal{C}\right\|_{L^{\bar{p}}} \frac{1}{R^{Q+2 k-s-t}}
$$

and since

$$
\left\|\tilde{u}_{n} \circ \mathcal{C}\right\|_{L^{\bar{p}}} \lesssim\left\|u_{n}\right\|_{L^{p^{*}}}^{p^{*}-1} \leq C,
$$

by letting first $n \rightarrow \infty$ then $R \rightarrow \infty$ we get

$$
\left\|\Lambda_{\mathcal{C}}^{\frac{1}{\bar{p}}} H_{k}^{\mathcal{L}_{2 k}}\left(\Lambda_{\mathcal{C}}^{\frac{1}{p^{*}}} u_{n} \circ \mathcal{C}, \eta \circ \mathcal{C}\right) \circ \mathcal{C}^{-1}\right\|_{H^{-k}}=o(1) .
$$

Now we move to estimating the term $\Lambda_{\mathcal{C}}^{\frac{1}{p^{*}}-\frac{1}{\bar{p}}} u_{n} \mathcal{L}_{2 k}(\eta \circ \mathcal{C}) \circ \mathcal{C}^{-1}$. Indeed, we have

$$
\left\|\Lambda_{\mathcal{C}}^{\frac{1}{p^{*}}} u_{n} \mathcal{L}_{2 k}(\eta \circ \mathcal{C}) \circ \mathcal{C}^{-1}\right\|_{L^{\bar{p}}} \lesssim\left\|u_{n}\right\|_{L^{2}}\left\|\Lambda_{\mathcal{C}}^{\frac{1}{p^{*}}} \mathcal{L}_{2 k}(\eta \circ \mathcal{C}) \circ \mathcal{C}^{-1}\right\|_{L^{\frac{Q}{k}}}
$$


and since $u_{n} \rightarrow 0$ in $L^{2}$ we have that

$$
\left\|\Lambda_{\mathcal{C}}^{\frac{1}{p^{*}}} u_{n} \mathcal{L}_{2 k}(\eta \circ \mathcal{C}) \circ \mathcal{C}^{-1}\right\|_{L^{\bar{p}}}=o(1) .
$$

Therefore, we have that $\|$ l.o.t $\|_{H^{-k}}=o(1)$. By the sub-elliptic regularity estimates we find

$$
\begin{aligned}
& \left\|\eta\left|u_{n}\right|\right\|_{H^{k}\left(B_{r}\left(\zeta_{0}\right)\right)} \lesssim\left\|\eta\left|u_{n}\right|^{p^{*}-2} u_{n}+\eta \delta_{n}\right\|_{H^{-k}\left(B_{r}\left(\zeta_{0}\right)\right)}+\| \text { l.o.t } \|_{H^{-k}\left(B_{r}\left(\zeta_{0}\right)\right)} \\
& \lesssim\left\|\eta\left|u_{n}\right|^{p^{*}-2} u_{n}\right\|_{H^{-k}\left(B_{r}\left(\zeta_{0}\right)\right)}+\left\|\eta \delta_{n}\right\|_{H^{-k}\left(B_{r}\left(\zeta_{0}\right)\right)}+o(1) .
\end{aligned}
$$

We estimate the first term in the inequality above as follows

$$
\begin{aligned}
\left\|\eta\left|u_{n}\right|^{p^{*}-2} u_{n}\right\|_{H^{-k}\left(B_{r}\left(\zeta_{0}\right)\right)} & \lesssim\left\|\eta\left|u_{n}\right|^{p^{*}-2} u_{n}\right\|_{L^{\frac{2 Q}{Q+2 k}}\left(B_{r}\left(\zeta_{0}\right)\right)} \\
& \lesssim\left\|u_{n}\right\|_{L^{p^{*}}\left(B_{r}\left(\zeta_{0}\right)\right)}^{\frac{4 k}{Q-2 k}}\left\|\eta u_{n}\right\|_{L^{p^{*}}\left(B_{r}\left(\zeta_{0}\right)\right)} \\
& \lesssim\left\|u_{n}\right\|_{L^{p^{*}}\left(B_{r}\left(\zeta_{0}\right)\right)}^{\frac{4 k}{Q-2 k}}\left\|\eta u_{n}\right\|_{H^{k}\left(B_{r}\left(\zeta_{0}\right)\right)} .
\end{aligned}
$$

Substituting the estimates above in (16) and using (14), we find

$$
\begin{aligned}
\left\|\eta u_{n}\right\|_{H^{k}\left(B_{r}\left(\zeta_{0}\right)\right)} & \lesssim\left\|u_{n}\right\|_{L^{p^{*}\left(B_{r}\left(\zeta_{0}\right)\right)}}^{\frac{4 k}{Q}}\left\|\eta u_{n}\right\|_{H^{k}\left(B_{r}\left(\zeta_{0}\right)\right)}+o(1) \\
& \lesssim \epsilon^{\frac{2 k}{Q}}\left\|\eta u_{n}\right\|_{H^{k}\left(B_{r}\left(\zeta_{0}\right)\right)}+o(1) .
\end{aligned}
$$

Now, we choose $\epsilon$ small enough to have $\left\|\eta u_{n}\right\|_{H^{k}\left(B_{r}\left(\zeta_{0}\right)\right)} \rightarrow 0$, leading to a contradiction to our assumptions.

Given $r>0$, We can define now the concentration function

$$
Q_{n}(r)=\sup _{\zeta \in S^{2 N+1}} \int_{B_{r}(\zeta)}\left|u_{n}\right|^{p^{*}} \mathrm{~d} v_{S}
$$

Since we are assuming that $u_{n}$ does not satisfies the (PS) condition, the Lemma above ensures the existence of a small enough $\epsilon_{0}>0$ such that $\Sigma_{\epsilon_{0}} \neq \emptyset$. Thus, for any fixed $\frac{\epsilon_{0}}{3}>\epsilon>0$, there exist a sequence of points $\zeta_{n} \in S^{2 N+1}$ and a sequence of radii $R_{n} \rightarrow 0$ such that

$$
Q_{n}\left(R_{n}\right)=\int_{B_{R_{n}}\left(\zeta_{n}\right)}\left|u_{n}\right|^{p^{*}} \mathrm{~d} v_{S}=\epsilon .
$$

Up to a subsequence, we can assume that $\zeta_{n} \rightarrow \zeta_{0} \in S^{2 N+1}$ as $n \rightarrow \infty$. Again, we fix a coordinate system in $\mathbb{C}^{N+1}$ so that $\zeta_{0}=(1,0, \ldots, 0)$ and denote by $-\zeta_{0}=(-1, \ldots, 0,0)$ the antipodal point of $\zeta_{0}$. We set

$$
\Omega=\mathcal{C}^{-1}\left(B_{1}\left(\zeta_{0}\right)\right) \subset \mathbb{H}^{N} .
$$

Clearly, for $n$ big all the balls $\mathcal{C}^{-1}\left(B_{R_{n}}\left(\zeta_{n}\right)\right)$ will be contained in $\Omega$. Hence, by means of the map $\mathcal{C}^{-1}$, the problem of characterizing (PS) sequences can be studied in $\mathbb{H}^{N}$, 
where the points $w_{n}=\mathcal{C}^{-1}\left(\zeta_{n}\right)$ accumulate at the point $0=\mathcal{C}^{-1}\left(\zeta_{0}\right)$ in the interior of the domain $\Omega$. Also, we define the map

$$
\rho_{n}: \mathbb{H}^{N} \rightarrow S^{2 N+1} \backslash\left\{-\zeta_{0}\right\}, \quad \rho_{n}(w)=\mathcal{C} \circ \tau_{w_{n}} \circ \delta_{R_{n}}(w)
$$

and the functions

$$
U_{n}=\Lambda_{\rho_{n}}^{\frac{Q-2 k}{2 Q}} u_{n} \circ \rho_{n},
$$

here $\Lambda_{\rho_{n}}$ is twice the absolute value of the Jacobian determinant of the map $\rho_{n}$. From now on we denote the preimage of a ball $B_{R R_{n}}\left(\zeta_{n}\right) \subset S^{2 N+1}$ with respect to the function $\rho_{n}$ by

$$
\mathcal{B}_{R}^{n}=\rho_{n}^{-1}\left(B_{R R_{n}}\left(\zeta_{n}\right)\right) .
$$

Notice that, for $n$ big, we can always assume $\zeta_{n} \in B_{\frac{1}{2}}\left(\zeta_{0}\right)$, hence $\mathcal{B}_{R}^{n}$ is well defined and $\mathcal{C}^{-1}\left(B_{R R_{n}}\left(\zeta_{n}\right)\right) \subset \Omega$ for every $R R_{n}<\frac{1}{2}$. Recalling the relation between $\mathcal{L}_{2 k}$ and $\mathcal{A}_{2 k}$ expressed in (6), we have

$$
\begin{aligned}
\int_{\mathcal{B}_{R}^{n}} U_{n} \mathcal{L}_{2 k} U_{n} \mathrm{~d} v_{H} & =\int_{B_{R R_{n}}\left(\zeta_{n}\right)} u_{n} \mathcal{A}_{2 k} u_{n} \mathrm{~d} v_{S}, \\
\int_{\mathcal{B}_{R}^{n}}\left|U_{n}\right|^{p^{*}} \mathrm{~d} v_{H} & =\int_{B_{R R_{n}}\left(\zeta_{n}\right)}\left|u_{n}\right|^{p^{*}} \mathrm{~d} v_{S} .
\end{aligned}
$$

In the sequel we will make use of the following relation obtained from inclusions (44)

$$
\mathcal{B}_{R}^{n}=\rho_{n}^{-1}\left(B_{R_{n} R}\left(\zeta_{n}\right)\right) \supset \delta_{R_{n}^{-1}} \circ \tau_{w_{n}^{-1}}\left(B_{\frac{R_{n} R}{2}}^{w_{n}}\right)=B_{\frac{R}{2}}^{0} .
$$

Also, we will use the notation

$$
\mathcal{B}_{R}^{0}=\mathcal{C}^{-1}\left(B_{R}\left(\zeta_{0}\right)\right)
$$

Lemma 3.5. Let us set $F_{n}=\mathcal{L}_{2 k} U_{n}-\left|U_{n}\right|^{p^{*}-2} U_{n}$, then for every $R>0$

$$
\sup \left\{\left\langle F_{n}, F\right\rangle_{H^{-k}, H^{k}}: \operatorname{supp}(F) \subset B_{R}^{0}, F \in H_{0}^{k}\left(B_{R}^{0}\right), \quad\|F\|_{H^{k}} \leq 1\right\} \rightarrow 0
$$

i.e.

$$
F_{n} \rightarrow 0 \quad \text { in } H_{\mathrm{loc}}^{-k}\left(\mathbb{H}^{N}\right) .
$$

Proof. Let us consider $n$ big enough to have $\left(6 R_{n}\right)^{-1} \geq R$, and $F \in H_{0}^{k}\left(B_{R}^{0}\right)$ such that $\operatorname{supp}(F) \subset B_{R}^{0}$ and $\|F\|_{H^{k}} \leq 1$. We have

$$
\begin{aligned}
\left\langle F_{n}, F\right\rangle_{H^{-k}, H^{k}} & =\int_{B_{\left(6 R_{n}\right)^{-1}}^{0}} F\left(\mathcal{L}_{2 k} U_{n}-\left|U_{n}\right|^{p^{*}-2} U_{n}\right) \mathrm{d} v_{H} \\
& \leq \int_{\mathcal{B}_{\left(3 R_{n}\right)^{-1}}} F\left(\mathcal{L}_{2 k} U_{n}-\left|U_{n}\right|^{p^{*}-2} U_{n}\right) \mathrm{d} v_{H} \\
& =\int_{\mathcal{B}_{\left(3 R_{n}\right)^{-1}}^{n}} \Lambda_{\rho_{n}}^{\frac{Q+2 k}{2 Q}} F\left(\mathcal{A}_{2 k} u_{n}-\left|u_{n}\right|^{p^{*}-2} u_{n}\right) \circ \rho_{n} \mathrm{~d} v_{H} \\
& =\int_{B_{\frac{1}{3}\left(\zeta_{n}\right)}}\left(\Lambda_{\rho_{n}}^{-\frac{Q-2 k}{2 Q}} F\right) \circ \sigma_{n}\left(\mathcal{A}_{2 k} u_{n}-\left|u_{n}\right|^{p^{*}-2} u_{n}\right) \mathrm{d} v_{S} .
\end{aligned}
$$


On the other hand, recalling ([6), we find

$$
\begin{aligned}
\left\|\left(\Lambda_{\rho_{n}}^{-\frac{Q-2 k}{2 Q}} F\right) \circ \sigma_{n}\right\|_{H^{k}} & =\int_{S^{2 N+1}} \Lambda_{\rho_{n}}^{-1}\left(F \mathcal{L}_{2 k} F\right) \circ \sigma_{n} \mathrm{~d} v_{S} \\
& =\int_{\mathbb{H}^{N}} F \mathcal{L}_{2 k} F \mathrm{~d} v_{H} \leq C,
\end{aligned}
$$

thus $\left\langle F_{n}, F\right\rangle_{H^{-k}, H^{k}} \rightarrow 0$.

Lemma 3.6. For $\epsilon>0$ small enough in (17), there exists $U_{\infty} \in D^{k}\left(\mathbb{H}^{N}\right)$ such that $U_{n} \rightarrow U_{\infty}$ in $H_{\mathrm{loc}}^{k}\left(\mathbb{H}^{N}\right)$ and

$$
\mathcal{L}_{2 k} U_{\infty}=\left|U_{\infty}\right|^{p^{*}-2} U_{\infty} \quad \text { on } \mathbb{H}^{N}
$$

Proof. The sequence $U_{n}$ is bounded in $H_{\mathrm{loc}}^{k}\left(\mathbb{H}^{N}\right)$, hence there exists $U_{\infty}$ such that, up to subsequence, $U_{n} \rightarrow U_{\infty}$ weakly in in $H_{\mathrm{loc}}^{k}\left(\mathbb{H}^{N}\right)$ and $U_{n} \rightarrow U_{\infty}$ strongly in $L_{\mathrm{loc}}^{p}\left(\mathbb{H}^{N}\right)$ for $1 \leq p<p^{*}$. From (18) we deduce

$$
\limsup _{n \rightarrow \infty} \int_{\mathcal{B}_{R}^{n}}\left|U_{n}\right|^{p^{*}} \mathrm{~d} v_{H} \leq \sup _{n \in \mathbb{N}} \int_{S^{2 N+1}}\left|u_{n}\right|^{p^{*}} \mathrm{~d} v_{S}<\infty
$$

so that $U_{\infty} \in L^{p^{*}}\left(\mathbb{H}^{N}\right)$. Moreover, by the same argument given after the proof of Lemma 3.1, we have that $U_{\infty}$ satisfies (3), hence

$$
\int_{\mathbb{H}^{N}} U_{\infty} \mathcal{L}_{2 k} U_{\infty} \mathrm{d} v_{H}<\infty
$$

It follows that $U_{\infty} \in D^{k}\left(\mathbb{H}^{N}\right)$.

In virtue of Lemma 3.2, we replace $U_{n}$ by $U_{n}-U_{\infty}$ so that, from now to the end of the proof, we can assume $U_{\infty}=0$. By (17) we have

$$
\int_{\mathcal{B}_{1}^{n}}\left|U_{n}\right|^{p^{*}} \mathrm{~d} v_{H}=\int_{B_{R_{n}}\left(\zeta_{n}\right)}\left|u_{n}\right|^{p^{*}} \mathrm{~d} v_{S}=\epsilon .
$$

Let $\beta \in C_{0}^{\infty}$, such that $\operatorname{supp}(\beta) \subset \mathcal{B}_{1}^{n}$ then

$$
\left\|\beta U_{n}\right\|_{H^{k}} \lesssim\left\|\mathcal{L}_{2 k}\left(\beta U_{n}\right)\right\|_{H^{-k}}+\left\|\beta U_{n}\right\|_{L^{2}} .
$$

Again, we use the fact that

$$
\begin{aligned}
\mathcal{L}_{2 k}\left(\beta U_{n}\right) & =\beta \mathcal{L}_{2 k}\left(U_{n}\right)+U_{n} \mathcal{L}_{2 k}(\beta)+H^{\mathcal{L}_{2 k}}\left(U_{n}, \beta\right) \\
& =\beta \mathcal{L}_{2 k}\left(U_{n}\right)+\text { l.o.t. }
\end{aligned}
$$

So first, we have that

$$
U_{n} \mathcal{L}_{2 k}(\beta)=U_{n} \chi_{B_{R}^{0}} \mathcal{L}_{2 k}(\beta)+U_{n} \chi_{\mathbb{H}^{N} \backslash B_{R}^{0}} \mathcal{L}_{2 k}(\beta)
$$


Therefore,

$$
\begin{aligned}
\left\|U_{n} \mathcal{L}_{2 k}(\beta)\right\|_{L^{\bar{p}}} & \lesssim\left\|U_{n}\right\|_{L^{2}\left(B_{R}^{0}\right)}\left\|\mathcal{L}_{2 k}(\beta)\right\|_{L^{\frac{Q}{k}}}+\left\|U_{n}\right\|_{L^{p^{*}}}\left\|\chi_{\mathbb{H}^{N} \backslash B_{R}^{0}} \mathcal{L}_{2 k}(\beta)\right\|_{L^{\frac{Q}{2 k}}} \\
& \lesssim\left\|U_{n}\right\|_{L^{2}\left(B_{R}^{0}\right)}+\frac{1}{R^{Q}}\left\|U_{n}\right\|_{L^{p^{*}}}
\end{aligned}
$$

Since $U_{n} \rightarrow 0$ in $L_{l o c}^{2}$ and $\left\|U_{n}\right\|_{L^{p^{*}}}$ is bounded, if we let $n \rightarrow \infty$ and then $R \rightarrow \infty$, we have that $\left\|U_{n} \mathcal{L}_{2 k}(\beta)\right\|_{L^{\bar{p}}}=o(1)$. Next, we move to the term $H^{\mathcal{L}_{2 k}}\left(\beta, U_{n}\right)$. Again, we have that from Lemma (commutator estimates),

$$
\left|H^{\mathcal{L}_{2 k}}\left(\beta, U_{n}\right)\right|(x) \lesssim \sum_{j=1}^{L} R_{2 k-s_{j, 1}-s_{j, 2}}\left(R_{s_{j, 1}}\left|\mathcal{L}_{2 k}\left(U_{n}\right)\right| R_{s_{j, 2}}\left|\mathcal{L}_{2 k}(\beta)\right|\right)(x)
$$

So we consider one term of the form $R_{2 k-s-t}\left(R_{t}\left|\mathcal{L}_{2 k}\left(U_{n}\right)\right| R_{s}\left|\mathcal{L}_{2 k}(\beta)\right|\right)(x)$. Using the same splitting as in (15), we have that

$$
\left\|H^{\mathcal{L}_{2 k}}\left(\beta, U_{n}\right)\right\|_{H^{-k}}=o(1),
$$

and thus $\|$ l.o.t $\|_{H^{-k}}=o(1)$. Clearly $\left\|\beta U_{n}\right\|_{L^{2}} \rightarrow 0$, and by Lemma 3.5 we know that $F_{n} \rightarrow 0$ in $H_{\text {loc }}^{-k}\left(\mathbb{H}^{N}\right)$, hence, we have

$$
\begin{aligned}
\left\|\mathcal{L}_{2 k}\left(\beta U_{n}\right)\right\|_{H^{-k}} & \leq \| \beta \mathcal{L}_{2 k} U_{n}+\text { l.o.t. } \|_{H^{-k}} \\
& \leq\left\|\beta\left(\left|U_{n}\right|^{p^{*}-2} U_{n}+F_{n}\right)\right\|_{H^{-k}}+o(1) \\
& \leq\left\|\beta\left|U_{n}\right|^{p^{*}-2} U_{n}\right\|_{H^{-k}}+o(1) .
\end{aligned}
$$

Therefore

$$
\left\|\beta U_{n}\right\|_{H^{k}} \lesssim\left\|\beta\left|U_{n}\right|^{p^{*}-2} U_{n}\right\|_{H^{-k}}+o(1)
$$

and

$$
\begin{aligned}
\left\|\beta U_{n}\right\|_{H^{k}} & \lesssim\left\|\beta\left|U_{n}\right|^{p^{*}-2} U_{n}\right\|_{L^{\bar{p}}\left(\mathcal{B}_{1}^{n}\right)}+o(1) \\
& \lesssim\left(\int_{\mathcal{B}_{1}^{n}}\left|U_{n}\right|^{p^{*}} \mathrm{~d} v_{H}\right)^{\frac{2 k}{Q}}\left\|\beta U_{n}\right\|_{L^{p^{*}}\left(\mathcal{B}_{1}^{n}\right)}+o(1) \\
& \lesssim \epsilon^{\frac{2 k}{Q}}\left\|\beta U_{n}\right\|_{L^{p^{*}}\left(\mathcal{B}_{1}^{n}\right)}+o(1) \\
& =o(1)
\end{aligned}
$$

as $n \rightarrow \infty$.

From the Lemma above and (21), it follows

$$
\int_{\mathcal{B}_{1}^{0}}\left|U_{\infty}\right|^{p^{*}} \mathrm{~d} v_{H}=\epsilon
$$


hence $U_{\infty} \neq 0$ is a solution to (3). We consider a cut off function $\gamma$ such that $\gamma \equiv 1$ on $B_{\frac{1}{4}}^{0}, \operatorname{supp}(\gamma) \subset B_{\frac{1}{2}}^{0}$, and we define $\beta=\gamma \circ \mathcal{C}^{-1}$. In virtue of the inclusions

$$
\mathcal{C}^{-1}\left(B_{\frac{1}{4}}\left(\zeta_{0}\right)\right) \subseteq B_{\frac{1}{4}}^{0} \subseteq \mathcal{C}^{-1}\left(B_{\frac{1}{2}}\left(\zeta_{0}\right)\right) \subseteq B_{\frac{1}{2}}^{0}
$$

the function $\beta$ is a cut off function such that $\beta \equiv 1$ on $B_{\frac{1}{4}}\left(\zeta_{0}\right)$ and $\operatorname{supp}(\beta) \subset B_{1}\left(\zeta_{0}\right)$, moreover for $n$ big enough we have

$$
\begin{aligned}
\operatorname{supp}\left(\beta \circ \rho_{n}\right)= & \operatorname{supp}\left(\gamma \circ \tau_{w_{n}} \circ \delta_{R_{n}}\right) \subseteq B_{\left(2 R_{n}\right)^{-1}}^{w_{n}} \subseteq B_{R_{n}^{-1}}^{0}, \\
& \beta \circ \rho_{n} \equiv 1 \quad \text { on } B_{\left(6 R_{n}\right)^{-1}}^{0} .
\end{aligned}
$$

We set

$$
v_{n}=\Lambda_{\sigma_{n}}^{\frac{Q-2 k}{2 Q}} \beta U_{\infty} \circ \sigma_{n}
$$

where $\Lambda_{\sigma_{n}}$ is half the absolute value of the Jacobian determinant of $\sigma_{n}$, and consider

$$
\bar{u}_{n}=u_{n}-v_{n} .
$$

For clarity sake, we recall here the definition of $u_{n}$ with respect to $U_{n}$

$$
u_{n}=\Lambda_{\sigma_{n}}^{\frac{Q-2 k}{2 Q}} U_{n} \circ \sigma_{n}
$$

We have then

Lemma 3.7. After taking a subsequence if necessary, we have

$$
\bar{u}_{n} \rightarrow 0 \quad \text { weakly in } H^{k}\left(S^{2 N+1}\right) .
$$

Proof. Since we have already proved that $u_{n} \rightarrow 0$, it suffices to show that $v_{n} \rightarrow 0$ weakly in $H^{k}\left(S^{2 N+1}\right)$. On the other hand, $v_{n}$ is bounded in $H^{k}\left(S^{2 N+1}\right)$ so that, after taking a subsequence if necessary, it converges to some limit; hence we only need to prove that the distributional limit is zero, i.e. it suffices to prove that for $f \in C^{\infty}$ it holds

$$
\int_{S^{2 N+1}} v_{n} f \mathrm{~d} v_{S} \rightarrow 0
$$

Let us fix $R>0$. We estimate the integral above, first on $B_{R_{n} R}\left(\zeta_{n}\right)$ and then on the exterior domain $S^{2 N+1} \backslash B_{R_{n} R}\left(\zeta_{n}\right)$, we have

$$
\begin{aligned}
\left|\int_{B_{R_{n} R}\left(\zeta_{n}\right)} v_{n} f \mathrm{~d} v_{S}\right| & =\left|\int_{B_{R_{n} R}\left(\zeta_{n}\right)} \Lambda_{\sigma_{n}}^{\frac{Q-2 k}{2 Q}} f \beta U_{\infty} \circ \sigma_{n} \mathrm{~d} v_{S}\right| \\
& =\left|\int_{\mathcal{B}_{R}^{n}} \Lambda_{\rho_{n}}^{\frac{Q+2 k}{2 Q}} U_{\infty}(f \beta) \circ \rho_{n} \mathrm{~d} v_{H}\right| \\
& \leq C R_{n}^{\frac{Q+2 k}{2}}\|f\|_{\infty}\left\|\Lambda_{\mathcal{C}}\right\|_{\infty}^{\frac{Q+2 k}{2 Q}} \int_{\mathcal{B}_{R}^{n}}\left|U_{\infty}\right| \mathrm{d} v_{H} .
\end{aligned}
$$


On the exterior domain, for $n$ big enough we find

$$
\begin{aligned}
\left|\int_{S^{2 N+1} \backslash B_{R_{n} R}\left(\zeta_{n}\right)} v_{n} f \mathrm{~d} v_{S}\right| & =\left|\int_{B_{1}\left(\zeta_{0}\right) \backslash B_{R_{n} R}\left(\zeta_{n}\right)} \Lambda_{\sigma_{n}}^{\frac{Q-2 k}{2 Q}} f \beta U_{\infty} \circ \sigma_{n} \mathrm{~d} v_{S}\right| \\
& \leq\left|\int_{B_{2 R_{n}^{n}}^{n} \backslash \mathcal{B}_{R}^{n}} \Lambda_{\rho_{n}}^{\frac{Q+2 k}{2 Q}} U_{\infty}(f \beta) \circ \rho_{n} \mathrm{~d} v_{H}\right| \\
& \leq C R_{n}^{\frac{Q+2 k}{2}}\|f\|_{\infty}\left\|\Lambda_{\mathcal{C}}\right\|_{\infty}^{\frac{Q+2 k}{2 Q}} \int_{B_{2 R_{n}^{-1}}^{0} \backslash B_{\frac{R}{2}}^{0}}\left|U_{\infty}\right| \mathrm{d} v_{H} . \\
& \leq C\|f\|_{\infty}\left\|\Lambda_{\mathcal{C}}\right\|_{\infty}^{\frac{Q+2 k}{2 Q}}\left(\int_{B_{2 R_{n}^{-1}}^{0} \backslash B_{\frac{R}{2}}^{0}}\left|U_{\infty}\right|^{p *} \mathrm{~d} v_{H}\right)^{\frac{1}{p^{*}}} .
\end{aligned}
$$

Then, the thesis follow letting $n \rightarrow \infty$ and then $R \rightarrow \infty$ in the following estimate

$$
\begin{aligned}
\left|\int_{S^{2 N+1}} v_{n} f \mathrm{~d} v_{S}\right| \lesssim & \|f\|_{\infty}\left\|\Lambda_{\mathcal{C}}\right\|_{\infty}^{\frac{Q+2 k}{2 Q}} R_{n}^{\frac{Q+2 k}{2}} \int_{\mathcal{B}_{R}^{n}}\left|U_{\infty}\right| \mathrm{d} v_{H} \\
& +\|f\|_{\infty}\left\|\Lambda_{\mathcal{C}}\right\|_{\infty}^{\frac{Q+2 k}{2 Q}}\left\|U_{\infty}\right\|_{L^{p^{*}}}\left(B_{2 R_{n}^{-1} \backslash B_{\frac{R}{2}}^{0}}\right)
\end{aligned}
$$

Lemma 3.8. We have

$$
d E\left(v_{n}\right) \rightarrow 0 \text { in } H^{-k}\left(S^{2 N+1}\right) \quad \text { and } \quad d E\left(\bar{u}_{n}\right) \rightarrow 0 \text { in } H^{-k}\left(S^{2 N+1}\right) .
$$

Proof. Let $f \in H^{k}\left(S^{2 N+1}\right)$ and $f_{n}=\mathcal{A}_{2 k} v_{n}-\left|v_{n}\right|^{p^{*}-2} v_{n}$. First we notice that

$$
\begin{aligned}
\mathcal{A}_{2 k}\left(v_{n}\right) & =\left(\Lambda_{\rho_{n}}^{-\frac{1}{\bar{p}}} \mathcal{L}_{2 k}\left(\Lambda_{\rho_{n}}^{\frac{1}{p^{*}}} v_{n} \circ \rho_{n}\right)\right) \circ \sigma_{n} \\
& =\left(\Lambda_{\rho_{n}}^{-\frac{1}{\bar{p}}} \mathcal{L}_{2 k}\left(\beta \circ \rho_{n} U_{\infty}\right)\right) \circ \sigma_{n} .
\end{aligned}
$$

Thus, we get,

$$
\begin{aligned}
\int_{S^{2 N+1}} f_{n} f \mathrm{~d} v_{S} & =\int_{S^{2 N+1}} f\left(\mathcal{A}_{2 k}\left(v_{n}\right)-\left|v_{n}\right|^{p^{*}-2} v_{n}\right) \mathrm{d} v_{S} \\
& =\int_{\mathbb{H}^{N}} \Lambda_{\rho_{n}}^{\frac{1}{p^{*}}} f \circ \rho_{n} \mathcal{L}_{2 k}\left(\beta \circ \rho_{n} U_{\infty}\right) \mathrm{d} v_{H}-\int_{S^{2 N+1}} f\left|v_{n}\right|^{p^{*}-2} v_{n} \mathrm{~d} v_{S} .
\end{aligned}
$$


Now notice that

$$
\begin{aligned}
\int_{\mathbb{H}^{N}} \Lambda_{\rho_{n}}^{\frac{1}{p^{*}}} f \circ \rho_{n} \mathcal{L}_{2 k}\left(\beta \circ \rho_{n} U_{\infty}\right) \mathrm{d} v_{H}= & \int_{\mathbb{H}^{N}} \Lambda_{\rho_{n}}^{\frac{1}{p^{*}}} f \circ \rho_{n} U_{\infty} \mathcal{L}_{2 k}\left(\beta \circ \rho_{n}\right) \mathrm{d} v_{H} \\
& +\int_{\mathbb{H}^{N}} \Lambda_{\rho_{n}}^{\frac{1}{p^{*}}}(f \beta) \circ \rho_{n} \mathcal{L}_{2 k}\left(U_{\infty}\right) \mathrm{d} v_{H} \\
& +\int_{\mathbb{H}^{N}} \Lambda_{\rho_{n}}^{\frac{1}{p^{*}}} f \circ \rho_{n} H^{\mathcal{L}_{2 k}}\left(U_{\infty}, \beta \circ \rho_{n}\right) \mathrm{d} v_{H} \\
= & \int_{S^{2 N+1}} f \beta\left(\Lambda_{\rho_{n}}^{-\frac{1}{\bar{p}}}\left|U_{\infty}\right|^{p^{*}-2} U_{\infty}\right) \circ \sigma_{n} \mathrm{~d} v_{S} \\
& +\int_{\mathbb{H}^{N}} \Lambda_{\rho_{n}}^{\frac{1}{p^{*}}} f \circ \rho_{n} U_{\infty} \mathcal{L}_{2 k}\left(\beta \circ \rho_{n}\right) \mathrm{d} v_{H} \\
& +\int_{\mathbb{H}^{N}} \Lambda_{\rho_{n}}^{\frac{1}{p^{*}}} f \circ \rho_{n} H^{\mathcal{L}_{2 k}}\left(U_{\infty}, \beta \circ \rho_{n}\right) \mathrm{d} v_{H} .
\end{aligned}
$$

Therefore, we have that

$$
\begin{aligned}
\int_{S^{2 N+1}} f_{n} f \mathrm{~d} v_{S}= & \int_{\mathbb{H}^{N}} \Lambda_{\rho_{n}}^{\frac{1}{p^{*}}} f \circ \rho_{n} U_{\infty} \mathcal{L}_{2 k}\left(\beta \circ \rho_{n}\right) \mathrm{d} v_{H} \\
& \quad+\int_{\mathbb{H}^{N}} \Lambda_{\rho_{n}}^{\frac{1}{p^{*}}} f \circ \rho_{n} H^{\mathcal{L}_{2 k}}\left(U_{\infty}, \beta \circ \rho_{n}\right) \mathrm{d} v_{H} \\
& \quad+\int_{S^{2 N+1}} f\left(\beta-\beta^{p^{*}-1}\right)\left(\Lambda_{\rho_{n}}^{-\frac{1}{\bar{p}}}\left|U_{\infty}\right|^{p^{*}-2} U_{\infty}\right) \circ \sigma_{n} \mathrm{~d} v_{S} \\
= & I_{1}+I_{2}+I_{3} .
\end{aligned}
$$

We estimate each of the three terms above separately. But first, we notice that

$$
\left\|\mathcal{L}_{2 k}\left(\beta \circ \rho_{n}\right)\right\|_{L^{p}}=R_{n}^{2 k-\frac{Q}{p}}\left\|\mathcal{L}_{2 k}(\gamma)\right\|_{L^{p}}
$$

In particular, if $p>\frac{Q}{2 k}$, then $\left\|\mathcal{L}_{2 k}\left(\beta \circ \rho_{n}\right)\right\|_{L^{p}} \rightarrow 0$. Now we have, for $R>1$,

$$
\begin{aligned}
\left|I_{1}\right| & =\left|\int_{\mathbb{H}^{N}} \Lambda_{\rho_{n}}^{\frac{1}{p^{*}}} f \circ \rho_{n} U_{\infty} \mathcal{L}_{2 k}\left(\beta \circ \rho_{n}\right) \mathrm{d} v_{H}\right| \\
& \leq\left\|\Lambda_{\rho_{n}}^{\frac{1}{p^{*}}} f \circ \rho_{n}\right\|_{L^{p^{*}}}\left\|U_{\infty} \mathcal{L}_{2 k}\left(\beta \circ \rho_{n}\right)\right\|_{L^{\bar{p}}} \\
& \leq\|f\|_{H^{k}}\left(\left\|U_{\infty}\right\|_{L^{q}}\left\|\mathcal{L}_{2 k}\left(\beta \circ \rho_{n}\right)\right\|_{L^{p}\left(B_{R}^{0}\right)}+\left\|U_{\infty}\right\|_{L^{p^{*}}\left(\mathbb{H}^{N} \backslash B_{R}^{0}\right)}\left\|\mathcal{L}_{2 k}\left(\beta \circ \rho_{n}\right)\right\|_{L^{\frac{e}{2 k}}}\right),
\end{aligned}
$$

where $\frac{1}{p}+\frac{1}{q}=\frac{1}{\bar{p}}$. Taking $p>\frac{Q}{2 k}$, we have for $R$ fixed that

$$
\left\|U_{\infty}\right\|_{L^{q}\left(B_{R}^{0}\right)}\left\|\mathcal{L}_{2 k}\left(\beta \circ \rho_{n}\right)\right\|_{L^{p}} \rightarrow 0 \text { as } n \rightarrow \infty .
$$

On the other hand, we have that

$$
\left\|U_{\infty}\right\|_{L^{p^{*}}\left(\mathbb{H}^{N} \backslash B_{R}^{0}\right)}\left\|\mathcal{L}_{2 k}\left(\beta \circ \rho_{n}\right)\right\|_{L^{\frac{Q}{2 k}}}=\left\|U_{\infty}\right\|_{L^{p^{*}}\left(\mathbb{H}^{N} \backslash B_{R}^{0}\right)}\left\|\mathcal{L}_{2 k}(\gamma)\right\|_{L^{\frac{Q}{2 k}}} \rightarrow 0 \text { as } R \rightarrow \infty
$$

Hence

$$
\left|I_{1}\right|=o(1)\|f\|_{H^{k}}
$$


We move now to the term $I_{2}$. First, we recall the following estimate for the Riesz potentials:

$$
\left\|R_{2 k-s-t}\left(R_{t}(u) R_{s}(v)\right)\right\|_{L^{p}} \lesssim\|u\|_{L^{q_{1}}}\|v\|_{L^{q_{2}}},
$$

for $\frac{1}{p}=\frac{1}{q_{1}}+\frac{1}{q_{2}}-\frac{2 k}{Q}$. In particular we have from Lemma (commutator estimates) and the previous estimates,

$$
\begin{aligned}
& \left\|H^{\mathcal{L}_{2 k}}\left(U_{\infty}, \beta \circ \rho_{n}\right)\right\|_{L^{\bar{p}}} \lesssim\left\|U_{\infty}^{p^{*}-1}\right\|_{L^{q}\left(B_{R}^{0}\right)}\left\|\mathcal{L}_{2 k}\left(\beta \circ \rho_{n}\right)\right\|_{L^{p}} \\
& +\left\|U_{\infty}^{p^{*}-1}\right\|_{L^{\bar{p}}\left(\mathbb{H}^{N} \backslash B_{R}^{0}\right)}\left\|\mathcal{L}_{2 k}\left(\beta \circ \rho_{n}\right)\right\|_{L^{\frac{Q}{2 k}}} \\
& \lesssim\left\|U_{\infty}\right\|_{L^{q\left(p^{*}-1\right)}\left(B_{R}^{0}\right)}^{p^{*}-1}\left\|\mathcal{L}_{2 k}\left(\beta \circ \rho_{n}\right)\right\|_{L^{p}} \\
& +\left\|U_{\infty}\right\|_{L^{p^{*}}\left(\mathbb{H}^{N} \backslash B_{R}^{0}\right)}^{p^{*}-1}\left\|\mathcal{L}_{2 k}(\gamma)\right\|_{L^{2 k}}
\end{aligned}
$$

where $\frac{1}{\bar{p}}=\frac{1}{p}+\frac{1}{q}-\frac{2 k}{Q}$. Hence, taking $p>\frac{2 k}{Q}$ and letting first $n \rightarrow 0$ then $R \rightarrow \infty$, we have that

$$
\left\|H^{\mathcal{L}_{2 k}}\left(U_{\infty}, \beta \circ \rho_{n}\right)\right\|_{L^{\bar{p}}}=o(1)
$$

In particular,

$$
\left|I_{2}\right| \leq\|f\|_{H^{k}}\left\|H^{\mathcal{L}_{2 k}}\left(U_{\infty}, \beta \circ \rho_{n}\right)\right\|_{L^{\bar{p}}}=o(1)\|f\|_{H^{k}} \quad \text { as } n \rightarrow \infty .
$$

Now we estimate the term $I_{3}$.

$$
\left|I_{3}\right| \lesssim\left\|\left(\beta-\beta^{p^{*}-1}\right) \circ \rho_{n}\left|U_{\infty}\right|^{p^{*}-2} U_{\infty}\right\|_{L^{\bar{p}}\left(\mathbb{H}^{N}\right)}\|f\|_{H^{k}}
$$

but $U_{\infty} \in D^{k}\left(\mathbb{H}^{N}\right)$ and

$$
\left\|\left(\beta-\beta^{p^{*}-1}\right) \circ \rho_{n}\left|U_{\infty}\right|^{p^{*}-2} U_{\infty}\right\|_{L^{\bar{p}}\left(\mathbb{H}^{N}\right)} \leq C\left\|U_{\infty}\right\|_{L^{p^{*}}\left(B_{2 R_{n}^{-1}}^{\frac{Q+2 k}{Q-2 k}} \backslash B_{\left(8 R_{n}\right)^{-1}}^{0}\right)}
$$

so that

$$
\left|I_{3}\right| \leq o(1)\|f\|_{H^{k}}
$$

Hence we have proved that $f_{n} \rightarrow 0$ in $H^{-k}\left(S^{2 N+1}\right)$. Now we turn to $d E\left(\bar{u}_{n}\right)$. Again, we consider $f \in H^{k}\left(S^{2 N+1}\right)$ and compute

$$
\begin{aligned}
\left\langle d E\left(\bar{u}_{n}\right), f\right\rangle= & \left\langle d E\left(u_{n}\right), f\right\rangle-\left\langle d E\left(v_{n}\right), f\right\rangle \\
& +\int_{S^{2 N+1}}\left(\left|u_{n}\right|^{p^{*}-2} u_{n}-\left|v_{n}\right|^{p^{*}-2} v_{n}-\left|\bar{u}_{n}\right|^{p^{*}-2} \bar{u}_{n}\right) f \mathrm{~d} v_{S} .
\end{aligned}
$$

We notice that, since $d E\left(u_{n}\right)$ and $d E\left(v_{n}\right)$ converge to zero in $H^{-k}\left(S^{2 N+1}\right)$, it suffices to show

$$
A_{n}=\left|u_{n}\right|^{p^{*}-2} u_{n}-\left|v_{n}\right|^{p^{*}-2} v_{n}-\left|\bar{u}_{n}\right|^{p^{*}-2} \bar{u}_{n} \rightarrow 0 \quad \text { in } H^{-k}\left(S^{2 N+1}\right) .
$$

In order to prove (26)), we will show $\left\|A_{n}\right\|_{L^{\bar{p}}\left(S^{2 N+1}\right)} \rightarrow 0$. Let us fix $R>0$. First we want to and obtain an estimate for $A_{n}$ in the exterior domain $D_{n}=S^{2 N+1} \backslash B_{R R_{n}}\left(\zeta_{n}\right)$ 
and then we will move to the interior of the ball $B_{R R_{n}}\left(\zeta_{n}\right)$. We write $u_{n}=\bar{u}_{n}+v_{n}$ in the definition of $A_{n}$ and we notice that for a big enough positive constant $C$ we have

$$
\begin{aligned}
\left|A_{n}\right| & =|| \bar{u}_{n}+\left.v_{n}\right|^{p^{*}-2}\left(\bar{u}_{n}+v_{n}\right)-\left|\bar{u}_{n}\right|^{p^{*}-2} \bar{u}_{n}-\left|v_{n}\right|^{p^{*}-2} v_{n} \mid \\
& \leq C\left(\left|\bar{u}_{n}\right|^{p^{*}-2}\left|v_{n}\right|+\left|v_{n}\right|^{p^{*}-2}\left|\bar{u}_{n}\right|\right) .
\end{aligned}
$$

Hence, by the Hölder inequality and recalling that $\operatorname{supp}\left(\beta \circ \rho_{n}\right) \subseteq B_{2 R_{n}^{-1}}^{0}$ and $B_{\frac{R}{2}}^{0} \subseteq \mathcal{B}_{R}^{n}$, we have

$$
\begin{aligned}
\left\|A_{n}\right\|_{L^{\bar{p}}\left(D_{n}\right)} \lesssim & \left(\left\|\left.\bar{u}_{n}\right|^{p^{*}-2} v_{n}\right\|_{L^{\bar{p}}\left(D_{n}\right)}+\left\|\bar{u}_{n}\left|v_{n}\right|^{p^{*}-2}\right\|_{L^{\bar{p}}\left(D_{n}\right)}\right) \\
& \lesssim\left(\left\|\bar{u}_{n}\right\|_{L^{p^{*}\left(D_{n}\right)}}^{\frac{4 k}{Q-2 k}}\left\|v_{n}\right\|_{L^{p^{*}}\left(D_{n}\right)}+\left\|\bar{u}_{n}\right\|_{L^{p^{*}\left(D_{n}\right)}}\left\|v_{n}\right\|_{L^{p^{*}\left(D_{n}\right)}}^{\frac{4 k}{Q-2 k}}\right) \\
\lesssim & \left\|\bar{u}_{n}\right\|_{L^{p^{*}\left(S^{2 N+1}\right)}}^{\frac{4 k}{Q-2 k}}\left\|U_{\infty}\right\|_{L^{p^{*}}}\left(B_{\left.2 R_{n}^{-1} \backslash B_{\frac{R}{2}}^{0}\right)}\right. \\
& +\left\|\bar{u}_{n}\right\|_{L^{p^{*}}\left(S^{2 N+1}\right)}\left\|U_{\infty}\right\|_{L^{p^{*}}}^{\frac{4 k}{Q-2 k}}\left(B_{2 R_{n}^{-1} \backslash B_{\frac{R}{2}}^{0}}^{0}\right) \\
= & o(1),
\end{aligned}
$$

as $R \rightarrow \infty$, uniformly in $n$. Similarly, in the interior of the ball $B_{R R_{n}}\left(\zeta_{n}\right)$ we find

$$
\begin{aligned}
& \left\|A_{n}\right\|_{L^{\bar{p}}\left(B_{R R_{n}}\left(\zeta_{n}\right)\right)} \lesssim\left(\left\|\left|\bar{u}_{n}\right|^{p^{*}-2} v_{n}\right\|_{L^{\bar{p}\left(B_{R R_{n}}\left(\zeta_{n}\right)\right)}}+\left\|\bar{u}_{n}\left|v_{n}\right|^{p^{*}-2}\right\|_{L^{\bar{p}}\left(B_{R R_{n}}\left(\zeta_{n}\right)\right)}\right) \\
& \lesssim\left\|\bar{u}_{n}\right\|_{L^{p^{*}\left(B_{R R_{n}}\left(\zeta_{n}\right)\right)}}^{\frac{4 k}{Q-2 k}}\left\|v_{n}\right\|_{L^{p^{*}}\left(B_{R R_{n}}\left(\zeta_{n}\right)\right)} \\
& +\left\|\bar{u}_{n}\right\|_{L^{p^{*}}\left(B_{R R_{n}}\left(\zeta_{n}\right)\right)}\left\|v_{n}\right\|_{L^{p^{*}\left(B_{R R_{n}}\left(\zeta_{n}\right)\right)}}^{\frac{4 k}{Q-2 k}} \\
& \lesssim\left\|U_{n}-\left(\beta \circ \rho_{n}\right) U_{\infty}\right\|_{L^{p^{*}}\left(\mathcal{B}_{R}^{n}\right)}^{\frac{4 k}{Q}}\left\|\left(\beta \circ \rho_{n}\right) U_{\infty}\right\|_{L^{p^{*}}\left(\mathcal{B}_{R}^{n}\right)} \\
& +\left\|U_{n}-\left(\beta \circ \rho_{n}\right) U_{\infty}\right\|_{L^{p^{*}}\left(\mathcal{B}_{R}^{n}\right)}\left\|\left(\beta \circ \rho_{n}\right) U_{\infty}\right\|_{L^{p^{*}}\left(\mathcal{B}_{R}^{n}\right)}^{\frac{4 k}{Q}} .
\end{aligned}
$$

Therefore, recalling the fact that $U_{n} \rightarrow U_{\infty}$ in $H_{\mathrm{loc}}^{k}\left(\mathbb{H}^{N}\right)$, we finally obtain

$$
\int_{B_{R R_{n}}\left(\zeta_{n}\right)}\left|A_{n}\right|^{\bar{p}} \mathrm{~d} v_{S} \rightarrow 0
$$

as desired.

Lemma 3.9. We have the following energy estimate

$$
E\left(\bar{u}_{n}\right)=E\left(u_{n}\right)-E_{\mathbb{H}}\left(U_{\infty}\right)+o(1) .
$$


Proof. We compute

$$
\begin{aligned}
E\left(\bar{u}_{n}\right)=\frac{1}{2} & \left(\int_{S^{2 N+1}} u_{n} \mathcal{A}_{2 k} u_{n} \mathrm{~d} v_{S}+\int_{S^{2 N+1}} v_{n} \mathcal{A}_{2 k} v_{n} \mathrm{~d} v_{S}-2 \int_{S^{2 N+1}} u_{n} \mathcal{A}_{2 k} v_{n} \mathrm{~d} v_{S}\right) \\
& -\frac{1}{p^{*}} \int_{S^{2 N+1}}\left|\bar{u}_{n}\right|^{p^{*}} \mathrm{~d} v_{S} .
\end{aligned}
$$

Let us estimate the second term in the identity above

$$
\begin{aligned}
\int_{S^{2 N+1}} v_{n} \mathcal{A}_{2 k} v_{n} \mathrm{~d} v_{S}= & \int_{\mathbb{H}^{N}} \beta \circ \rho_{n} U_{\infty} \mathcal{L}_{2 k}\left(\beta \circ \rho_{n} U_{\infty}\right) \mathrm{d} v_{H} \\
= & \int_{\mathbb{H}^{N}} \beta^{2} \circ \rho_{n} U_{\infty} \mathcal{L}_{2 k} U_{\infty} \mathrm{d} v_{H}+\int_{\mathbb{H}^{N}} \beta \circ \rho_{n} U_{\infty}^{2} \mathcal{L}_{2 k}\left(\beta \circ \rho_{n}\right) \mathrm{d} v_{H} \\
& +\int_{\mathbb{H}^{N}} \beta \circ \rho_{n} U_{\infty} H^{\mathcal{L}_{2 k}}\left(U_{\infty}, \beta \circ \rho_{n}\right) \mathrm{d} v_{H} \\
= & I_{1}+I_{2}+I_{3} .
\end{aligned}
$$

Clearly,

$$
I_{1}=\int_{\mathbb{H}^{N}} U_{\infty} \mathcal{L}_{2 k} U_{\infty} \mathrm{d} v_{H}+o(1)
$$

and using the same argument as in Lemma 3.6.

$$
\begin{aligned}
& \left|I_{2}\right|=\left|\int_{\mathbb{H}^{N}} \beta \circ \rho_{n} U_{\infty}^{2} \mathcal{L}_{2 k}\left(\beta \circ \rho_{n}\right) \mathrm{d} v_{H}\right| \\
& \lesssim\left\|U_{\infty}^{2}\right\|_{L^{q}\left(B_{R}^{0}\right)}\left\|\mathcal{L}_{2 k}\left(\beta \circ \rho_{n}\right)\right\|_{L^{p}}+\left\|U_{\infty}^{2}\right\|_{L^{\frac{Q}{Q-2 k}\left(\mathbb{H}^{N} \backslash B_{R}^{0}\right)}}\left\|\mathcal{L}_{2 k}\left(\beta \circ \rho_{n}\right)\right\|_{L^{\frac{Q}{2 k}}} \\
& \lesssim\left\|U_{\infty}\right\|_{L^{2 q}\left(B_{R}^{0}\right)}^{2}\left\|\mathcal{L}_{2 k}\left(\beta \circ \rho_{n}\right)\right\|_{L^{p}}+\left\|U_{\infty}\right\|_{L^{p^{*}}\left(\mathbb{H}^{N} \backslash B_{R}^{0}\right)}^{2}\left\|\mathcal{L}_{2 k}(\gamma)\right\|_{L^{2 k}}
\end{aligned}
$$

for $\frac{1}{p}+\frac{1}{q}=1$, taking $p>\frac{Q}{2 k}$ and letting $n \rightarrow \infty$ then $R \rightarrow \infty$, we have that

$$
I_{2}=o(1),
$$

as $n \rightarrow \infty$. Now for $I_{3}$, using (25), we have

$$
\left|I_{3}\right| \lesssim\left\|U_{\infty}\right\|_{L^{p^{*}}}\left\|H^{\mathcal{L}_{2 k}}\left(U_{\infty}, \beta \circ \rho_{n}\right)\right\|_{L^{\bar{p}}}=o(1) .
$$

Combining the estimates of $I_{1}, I_{2}$ and $I_{3}$, we find

$$
\int_{S^{2 N+1}} v_{n} \mathcal{A}_{2 k} v_{n} \mathrm{~d} v_{S}=\int_{\mathbb{H}^{N}} U_{\infty} \mathcal{L}_{2 k} U_{\infty} \mathrm{d} v_{H}+o(1)
$$

Now we estimate the third term in (27)

$$
\begin{aligned}
\int_{S^{2 N+1}} u_{n} \mathcal{A}_{2 k} v_{n} \mathrm{~d} v_{S}= & \int_{\mathbb{H}^{N}} U_{n} \mathcal{L}_{2 k}\left(\beta \circ \rho_{n} U_{\infty}\right) \mathrm{d} v_{H} \\
= & \int_{\mathbb{H}^{N}} \beta \circ \rho_{n} U_{n} \mathcal{L}_{2 k} U_{\infty} \mathrm{d} v_{H}+\int_{\mathbb{H}^{N}} U_{n} U_{\infty} \mathcal{L}_{2 k}\left(\beta \circ \rho_{n}\right) \mathrm{d} v_{H} \\
& +\int_{\mathbb{H}^{N}} U_{n} H^{\mathcal{L}_{2 k}}\left(U_{\infty}, \beta \circ \rho_{n}\right) \mathrm{d} v_{H} \\
= & I_{4}+I_{5}+I_{6} .
\end{aligned}
$$


Let us fix $R>0$ and define $B_{n}=B_{2 R_{n}^{-1}}^{0} \backslash B_{R}^{0}$. For $n$ big enough to have $\beta \circ \rho_{n} \equiv 1$ on $B_{\left(6 R_{n}\right)^{-1}}^{0} \supset B_{R}^{0}$, we get

$$
I_{4}=\int_{B_{R}^{0}} U_{n} \mathcal{L}_{2 k} U_{\infty} \mathrm{d} v_{H}+\int_{B_{n}} \beta \circ \rho_{n} U_{n} \mathcal{L}_{2 k} U_{\infty} \mathrm{d} v_{H},
$$

and we estimate the second term in the identity above by

$$
\left|\int_{B_{n}} \beta \circ \rho_{n} U_{n} \mathcal{L}_{2 k} U_{\infty} \mathrm{d} v_{H}\right| \leq C\left\|U_{n}\right\|_{L^{p^{*}}\left(B_{2 R_{n}^{-1}}^{0}\right)}\left\|\mathcal{L}_{2 k} U_{\infty}\right\|_{L^{\bar{p}}\left(B_{n}\right)}=o(1),
$$

as $R \rightarrow \infty$ uniformly in $n$. Hence, since $U_{n} \rightarrow U_{\infty}$ in $H_{\text {loc }}^{k}\left(\mathbb{H}^{N}\right)$

$$
I_{4}=\int_{B_{R}^{0}} U_{\infty} \mathcal{L}_{2 k} U_{\infty} \mathrm{d} v_{H}+o(1) .
$$

Let us turn the attention to $I_{5}$

$$
\begin{aligned}
& \left|I_{5}\right|=\left|\int_{\mathbb{H}^{N}} U_{n} U_{\infty} \mathcal{L}_{2 k}\left(\beta \circ \rho_{n}\right) \mathrm{d} v_{H}\right|
\end{aligned}
$$

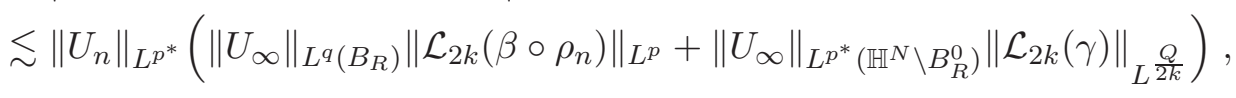

where $\frac{1}{p}+\frac{1}{q}=\frac{1}{\bar{p}}$. Once again, if we take $p>\frac{Q}{2 k}$, and let $n \rightarrow \infty$ then $R \rightarrow \infty$ we get

$$
I_{5}=o(1) \text {. }
$$

Also,

$$
\left|I_{6}\right| \leq\left\|U_{n}\right\|_{L^{p^{*}}}\left\|H^{\mathcal{L}_{2 k}}\left(U_{\infty}, \beta \circ \rho_{n}\right)\right\|_{L^{\bar{p}}}=o(1) .
$$

Combining the estimates for $I_{4}, I_{5}$ and $I_{6}$ we get

$$
\int_{S^{2 N+1}} u_{n} \mathcal{A}_{2 k} v_{n} \mathrm{~d} v_{S}=\int_{B_{R}^{0}} U_{\infty} \mathcal{L}_{2 k} U_{\infty} \mathrm{d} v_{H}+o(1) .
$$

We consider now the last term in (27). We are going to show that

$$
\int_{S^{2 N+1}}\left|\bar{u}_{n}\right|^{p^{*}} \mathrm{~d} v_{S}=\int_{S^{2 N+1}}\left|u_{n}\right|^{p^{*}} \mathrm{~d} v_{S}-\int_{\mathbb{H}^{N}}\left|U_{\infty}\right|^{p^{*}} \mathrm{~d} v_{H}+o(1) \quad \text { as } n \rightarrow \infty .
$$

Hence, using (28), (29) and (30) to estimate the right hand side of (27) we get

$$
E\left(\bar{u}_{n}\right)=E\left(u_{n}\right)-E_{\mathbb{H}}\left(U_{\infty}\right)+o(1),
$$

as desired. Before proving (30) we make a few observations. Let us fix $R>0$ and define $D_{n}=S^{2 N+1} \backslash B_{R R_{n}}\left(\zeta_{n}\right)$. First, we notice that for $n$ big enough to have $\beta \circ \rho_{n} \equiv 1$ on $B_{R}^{0}$ we find

$$
\int_{B_{R R_{n}}\left(\zeta_{n}\right)}\left|\bar{u}_{n}\right|^{p^{*}} \mathrm{~d} v_{S}=\int_{\mathcal{B}_{R}^{n}}\left|U_{n}-U_{\infty}\right|^{p^{*}} \mathrm{~d} v_{H}=o(1),
$$


as $n \rightarrow \infty$, since by Lemma $3.6 U_{n} \rightarrow U_{\infty}$ in $H_{\text {loc }}^{k}\left(\mathbb{H}^{N}\right)$. Also,

$$
\int_{D_{n}}\left|v_{n}\right|^{p^{*}} \mathrm{~d} v_{S}=\int_{B_{2 R_{n}^{-1}}^{0} \backslash \mathcal{B}_{R}^{n}}\left|\beta \circ \rho_{n} U_{\infty}\right|^{p^{*}} \mathrm{~d} v_{H} \leq C \int_{\mathbb{H}^{N} \backslash B_{\frac{R}{2}}^{0}}\left|U_{\infty}\right|^{p^{*}} \mathrm{~d} v_{H}=o(1),
$$

as $R \rightarrow \infty$ uniformly in $n$. Moreover

$$
\int_{D_{n}}\left|\bar{u}_{n}\right|^{p^{*}} \mathrm{~d} v_{S}=\int_{D_{n}}\left|u_{n}\right|^{p^{*}} \mathrm{~d} v_{S}-\int_{D_{n}}\left|v_{n}\right|^{p^{*}} \mathrm{~d} v_{S}+o(1)
$$

as $R \rightarrow \infty$ uniformly in $n$. Indeed, for a suitable constant $C$, independent of $n$ we have

$$
\left.\int_{D_{n}}|| u_{n}\right|^{p^{*}}-\left|\bar{u}_{n}\right|^{p^{*}}-\left.\left|v_{n}\right|^{p^{*}}\left|\mathrm{~d} v_{S} \leq C \int_{D_{n}}\right| \bar{u}_{n}\right|^{p^{*}-1}\left|v_{n}\right| \mathrm{d} v_{S}+C \int_{D_{n}}\left|v_{n}\right|^{p^{*}-1}\left|\bar{u}_{n}\right| \mathrm{d} v_{S}
$$

but

$$
\begin{aligned}
\left.\int_{D_{n}}\left|\bar{u}_{n}\right|\right|^{p^{*}-1}\left|v_{n}\right| \mathrm{d} v_{S} & =\int_{\mathbb{H}^{N} \backslash \mathcal{B}_{R}^{n}}\left|U_{n}-\beta \circ \rho_{n} U_{\infty}\right|^{p^{*}-1}\left|\beta \circ \rho_{n} U_{\infty}\right| \mathrm{d} v_{H} \\
& \lesssim \int_{B_{n}}\left|U_{n}-\beta \circ \rho_{n} U_{\infty}\right|^{p^{*}-1}\left|U_{\infty}\right| \mathrm{d} v_{H} \\
& \lesssim\left\|U_{n}-\beta \circ \rho_{n} U_{\infty}\right\|_{L^{p^{*}}\left(B_{n}\right)}^{\frac{Q+2 k}{Q-2 k}}\left\|U_{\infty}\right\|_{L^{p^{*}}\left(B_{n}\right)} \\
& \lesssim\left\|U_{\infty}\right\|_{L^{p^{*}}\left(B_{n}\right)} \\
& =o(1)
\end{aligned}
$$

as $R \rightarrow \infty$ uniformly in $n$. Similarly

$$
\begin{aligned}
\int_{D_{n}}\left|v_{n}\right|^{p^{*}-1}\left|\bar{u}_{n}\right| \mathrm{d} v_{S} & \lesssim \int_{\mathbb{H}^{N} \backslash B_{R}^{0}}\left|\beta \circ \rho_{n} U_{\infty}\right|^{p^{*}-1}\left|U_{n}-\beta \circ \rho_{n} U_{\infty}\right| \mathrm{d} v_{H} \\
& \lesssim\left\|U_{\infty}\right\|_{L^{p^{*}\left(B_{n}\right)}}^{\frac{Q+2 k}{Q}}\left\|U_{n}-\beta \circ \rho_{n} U_{\infty}\right\|_{L^{p^{*}}\left(B_{n}\right)} \\
& \lesssim\left\|U_{\infty}\right\|_{L^{p^{*}}\left(B_{n}\right)} \\
& =o(1)
\end{aligned}
$$


as $R \rightarrow \infty$ uniformly in $n$, proving (33). We are ready now to prove (30):

$$
\begin{aligned}
\int_{S^{2 N+1}}\left|\bar{u}_{n}\right|^{p^{*}} \mathrm{~d} v_{S} & =\int_{B_{R R_{n}}\left(\zeta_{n}\right)}\left|\bar{u}_{n}\right|^{p^{*}} \mathrm{~d} v_{S}+\int_{D_{n}}\left|\bar{u}_{n}\right|^{p^{*}} \mathrm{~d} v_{S} \\
& \stackrel{(31)}{=} \int_{D_{n}}\left|\bar{u}_{n}\right|^{p^{*}} \mathrm{~d} v_{S}+o(1) \\
& \stackrel{(33)}{=} \int_{D_{n}}\left|u_{n}\right|^{p^{*}} \mathrm{~d} v_{S}-\int_{D_{n}}\left|v_{n}\right|^{p^{*}} \mathrm{~d} v_{S}+o(1) \\
& \stackrel{(32)}{=} \int_{D_{n}}\left|u_{n}\right|^{p^{*}} \mathrm{~d} v_{S}+o(1) \\
& =\int_{S^{2 N+1}}\left|u_{n}\right|^{p^{*}} \mathrm{~d} v_{S}-\int_{B_{R R_{n}}\left(\zeta_{n}\right)}\left|u_{n}\right|^{p^{*}} \mathrm{~d} v_{S}+o(1) \\
& =\int_{S^{2 N+1}}\left|u_{n}\right|^{p^{*}} \mathrm{~d} v_{S}-\int_{\mathcal{B}_{R}^{n}}\left|U_{n}\right|^{p^{*}} \mathrm{~d} v_{H}+o(1) .
\end{aligned}
$$

Now, recalling that $\int_{\mathcal{B}_{R}^{n}}\left|U_{n}\right|^{p^{*}} \mathrm{~d} v_{H} \rightarrow \int_{\mathcal{B}_{R}^{0}}\left|U_{\infty}\right|^{p^{*}} \mathrm{~d} v_{H}$ for any $R$ as $n \rightarrow \infty$ we finally get (30).

Remark 3.1. Let $\omega$ be defined by (10). Let us explicitly recall that the functions

$$
\omega_{\lambda, \xi}=\lambda^{\frac{2 k-Q}{2}} \omega \circ \delta_{\lambda^{-1}} \circ \tau_{\xi^{-1}}, \quad \lambda>0, \xi \in \mathbb{H}^{N},
$$

are solutions to (3) which have all the same energy

$$
C_{E}:=E_{\mathbb{H}}\left(\omega_{\lambda, \xi}\right)=\frac{k}{Q} C_{S}^{-\frac{Q}{2 k}}>0, \quad \forall \lambda>0 \text { and } \xi \in \mathbb{H}^{N} .
$$

where $C_{S}$ is the Sobolev constant in (8). In particular they are the only ones with this energy ([9]).

Now we conclude the proof of the main result.

proof of Theorem 1.1. We define

$$
u_{n}^{1}:=u_{n}-u_{\infty}
$$

and by Lemma 3.2 we have

$$
E\left(u_{n}^{1}\right)=E\left(u_{n}\right)-E\left(u_{\infty}\right)+o(1) .
$$

By the procedure described above, we find a sequence of points $\zeta_{n}^{1}$ converging to a concentration point $\zeta^{1} \in S^{2 N+1}$, a sequence of radii $R_{n}^{1}$ converging to zero, a solution $U_{\infty}^{1}$ to equation (3) and a sequence $v_{n}^{1}$ defined as in (23); therefore we set:

$$
u_{n}^{2}:=u_{n}^{1}-v_{n}^{1}=u_{n}-u_{\infty}-v_{n}^{1} .
$$


By Lemma 3.9, we get

$$
E\left(u_{n}^{2}\right)=E\left(u_{n}^{1}\right)-E_{\mathbb{H}}\left(U_{\infty}^{1}\right)+o(1)=E\left(u_{n}\right)-E\left(u_{\infty}\right)-E_{\mathbb{H}}\left(U_{\infty}^{1}\right)+o(1) .
$$

Now we iteratively apply this procedure obtaining

$$
u_{n}^{m}=u_{n}-u_{\infty}-\sum_{l=1}^{m-1} v_{n}^{l}
$$

and

$$
E\left(u_{n}^{m}\right)=E\left(u_{n}\right)-E\left(u_{\infty}\right)-\sum_{l=1}^{m-1} E_{\mathbb{H}}\left(U_{\infty}^{l}\right)+o(1) .
$$

Since $E_{\mathbb{H}}\left(U_{\infty}^{l}\right) \geq C_{E}$ for every $l=1 \ldots m$, we stop the process when $c-m C_{E}<C_{E}$. Indeed, by Lemma 3.3. (PS) sequences at levels strictly below $\frac{k}{Q} C_{S}^{-\frac{Q}{2 k}}$ converge strongly in $H^{k}\left(S^{2 N+1}\right)$, and this concludes the proof.

\section{Existence of infinitely many solutions}

In this section we will prove the existence of infinitely many solutions of (3) proceeding as in [19]. The key idea is to find a suitable subspace of the space of variations for the functional we are interested in, on which it is straightforward to perform the following minimax argument by Ambrosetti and Rabinowitz (see [1, Theorems 3.13 and 3.14]).

Lemma 4.1. Let $X$ be a closed infinite dimensional subspace of $H^{k}\left(S^{2 N+1}\right)$. Assume that $E_{\left.\right|_{X}}$, the restriction of $E$ on $X$, satisfies the Palais-Smale compactness condition on $X$. Then, there exists a sequence $u_{n}$ of critical points for $E_{\left.\right|_{X}}$ such that

$$
\int_{S^{2 N+1}}\left|u_{n}\right|^{p^{*}} \mathrm{~d} v_{S} \rightarrow \infty \quad \text { as } n \rightarrow \infty .
$$

Let us start by fixing some notations. We denote by $\mathbb{O}(2 N+2)$, the group of $(2 N+$ $2) \times(2 N+2)$ orthogonal matrices, and

$$
\mathbb{U}(N+1)=\{g \in \mathbb{O}(2 N+2): g J=J g\},
$$

where

$$
J=\left(\begin{array}{cc}
0 & -I_{N+1} \\
I_{N+1} & 0
\end{array}\right)
$$

Since the elements of $\mathbb{U}(N+1)$ define isometries on $S^{2 N+1}$ and $\mathcal{A}_{2 k}$ is an intertwining operator (i.e. satisfies (5)), it follows that the functional $E$ is invariant under the action of $\mathbb{U}(N+1)$ :

$$
E(u)=E(u \circ g), \quad \text { for all } g \in \mathbb{U}(N+1),
$$

(for a detailed proof see for instance [13]). For a subgroup $G$ of $\mathbb{U}(N+1)$ we define

$$
X_{G}=\left\{u \in H^{k}\left(S^{2 N+1}\right): u \circ g=u, \forall g \in G\right\} .
$$


Lemma 4.2. Let $G$ be a subgroup of $\mathbb{U}(N+1)$ such that for any $\zeta_{0} \in S^{2 N+1}$ the $G$ orbit of $\zeta_{0}$ has at least one accumulation point. Then, $E_{\left.\right|_{X_{G}}}$, the restriction of $E$ to $X_{G}$, satisfies the Palais-Smale condition.

Proof. Let $u_{n}$ be a (PS) sequence for $E_{\left.\right|_{X_{G}}}$ at level $c$. By contradiction, we suppose that $u_{n}$ does not admit a converging subsequence in $X_{G}$. Hence, by the classification of (PS) sequence given in Theorem 1.1, we deduce that the set of concentration points

$$
\Theta=\left\{\zeta^{l} \in S^{2 N+1}: 1 \leq l \leq m\right\}
$$

is discrete, finite and non-empty. Here we have adopted the same notation used in Theorem 1.1. Let $\zeta_{0} \in \Theta$. Then, since the (PS) sequence $u_{n}$ is invariant under the action of $G$, if $g_{i}$ with $i=1, \ldots, j$, are $j$ fixed elements in $G$, we have that also $\zeta^{i}=g_{i} \zeta_{0}$ are concentration points belonging to $\Theta$. Again, by Theorem 1.1 and recalling Remark 3.1 we have

$$
c=\lim _{n \rightarrow \infty} E\left(u_{n}\right)=E\left(u_{\infty}\right)+\sum_{i=1}^{j} E_{\mathbb{H}^{N}}\left(U_{\infty}^{i}\right) \geq E\left(u_{\infty}\right)+j \frac{k}{Q} C_{S}^{-\frac{Q}{2 k}} .
$$

On the other hand, by assumption, the $G$-orbit of $\zeta_{0}$ has an accumulation point, therefore $\Theta$ contains infinitely many concentration points of the type $\zeta_{i}=g_{i} \zeta_{0}$. Hence, letting $j \rightarrow+\infty$ in (35) we reach a contradiction.

We recall that quite a few examples of infinite dimensional subgroups of $\mathbb{U}(N+1)$ satisfying hypotheses of Lemma 4.2 are provided in [19] and [13]. Now we prove our result:

Proof of Theorem 1.2. Let $G$ be a subgroup of $\mathbb{U}(N+1)$ such that $X_{G}$ is an infinite dimensional vector space and suppose that for each $\zeta_{0} \in S^{2 N+1}$ the $G$-orbit of $\zeta_{0}$ contains at least one accumulation point. By Lemma 4.2, $E_{\left.\right|_{X_{G}}}$ satisfies the PalaisSmale condition, therefore Lemma 4.1 allows to perform a minimax argument ensuring the existence of a sequence of critical points $u_{n}$ in $X_{G}$ for $E_{\left.\right|_{X_{G}}}$ such that

$$
\int_{S^{2 N+1}}\left|u_{n}\right|^{p^{*}} \mathrm{~d} v_{S} \rightarrow \infty \quad \text { as } n \rightarrow \infty
$$

Now, since the functional $E$ is invariant under the action of $G$, by the Principle of Symmetric Criticality (see [20]), we have that any critical point of $E_{\left.\right|_{X_{G}}}$ is also a critical point for $E$. Moreover, to each $u_{n}$, solution to (2), corresponds a solution $U_{n}=\Lambda_{\mathcal{C}}^{\frac{1}{p^{*}}} u_{n} \circ \mathcal{C}$ to (3) and (36) implies

$$
\begin{aligned}
\int_{\mathbb{H}^{N}} U_{n} \mathcal{L}_{2 k} U_{n} \mathrm{~d} v_{H} & =\int_{\mathbb{H}^{N}} \Lambda_{\mathcal{C}}^{\frac{1}{p^{*}}} u_{n} \circ \mathcal{C} \mathcal{L}_{2 k}\left(\Lambda_{\mathcal{C}}^{\frac{1}{p^{*}}} u_{n} \circ \mathcal{C}\right) \mathrm{d} v_{H} \\
& =\int_{S^{2 N+1}} u_{n} \mathcal{A}_{2 k} u_{n} \mathrm{~d} v_{S} \\
& =\int_{S^{2 N+1}}\left|u_{n}\right|^{p^{*}} \mathrm{~d} v_{S} \rightarrow \infty \quad \text { as } n \rightarrow \infty .
\end{aligned}
$$


But all the solutions to (3) of the type $\omega_{\lambda, \xi}$ have the same energy $\frac{k}{Q} C_{S}^{-\frac{Q}{2 k}}$ (see Remark 3.1), consequently

$$
\int_{\mathbb{H}^{N}} \omega_{\lambda, \xi} \mathcal{L}_{2 k} \omega_{\lambda, \xi} \mathrm{d} v_{H}=\left(\frac{1}{2}-\frac{1}{p^{*}}\right)^{-1} E_{\mathbb{H}}\left(\omega_{\lambda, \xi}\right)=\left(\frac{1}{2}-\frac{1}{p^{*}}\right)^{-1} \frac{k}{Q} C_{S}^{-\frac{Q}{2 k}} .
$$

So, it is clear that in the sequence $U_{n}$ (and hence in the sequence $u_{n}$ ) there are infinitely many solutions of (3) (or equivalently of (2) ), distinct from $\omega_{\lambda, \xi}$.

\section{A Appendix}

We recall here some definitions and properties for the Riesz potentials on Carnot groups. So let $\mathbb{G}$ be a Carnot group of homogeneous dimension $Q$ and $\Delta_{b}$ its sub-Laplacian, then we have

Theorem A.1 (9]). Let $0<\alpha<Q$ and consider $h(t, x)$ the fundamental solution of the operator $-\Delta_{b}+\frac{\partial}{\partial t}$, then the integral

$$
R_{\alpha}(x)=\frac{1}{\Gamma\left(\frac{\alpha}{2}\right)} \int_{0}^{\infty} t^{\frac{\alpha}{2}-1} h(t, x) d t
$$

converges absolutely and it satisfies the following properties:

- $R_{\alpha}$ is a kernel of type $\alpha$. In particular it is homogeneous of degree $\alpha-Q$

- $R_{2}$ is the fundamental solution of $-\Delta_{b}$

- $R_{\alpha} * R_{\beta}=R_{\alpha+\beta}$ for $\alpha$ and $\beta>0$ and $\alpha+\beta<Q$.

- For $f \in L^{p}(\mathbb{G})$ and $1<p<\infty$, we have that

$$
\left(-\Delta_{b}\right)^{-\frac{\alpha}{2}} f=f * R_{\alpha} .
$$

In this paper, we used the convention

$$
R_{\alpha} f:=\left(-\Delta_{b}\right)^{-\frac{\alpha}{2}} f=f * R_{\alpha} .
$$

From the integral form of $R_{\alpha}$ one has

$$
R_{\alpha}(x) \approx|x|^{-Q+\alpha}
$$

and $\rho(x):=\left(R_{\alpha}(x)\right)^{\frac{1}{\alpha-Q}}$ defines a $\mathbb{G}$-homogeneous norm, smooth away from the origin and it induces a quasi-distance that is equivalent to the left-invariant CarnotCaratheodory distance. In a similar way, one can define the function $\tilde{R}_{\alpha}$, introduced in [6], for $\alpha<0$ and $\alpha \notin\{0,-2,-4, \ldots\}$ by

$$
\tilde{R}_{\alpha}(x)=\frac{\frac{\alpha}{2}}{\Gamma\left(\frac{\alpha}{2}\right)} \int_{0}^{\infty} t^{\frac{\alpha}{2}-1} h(t, x) d t
$$


Again, it is easy to see that $\tilde{R}_{\alpha}$ is $\mathbb{G}$-homogeneous of degree $\alpha-Q$ and

$$
\tilde{R}_{\alpha}(x) \approx|x|^{\alpha-Q}
$$

Using this function, it is possible to define another representation for the fractional sub-Laplacian, which we use in the proofs of our results.

Theorem A.2 ([6]). If $u$ is a Schwartz function on $\mathbb{G}$, then for $0<\alpha<2$ one has

$$
\left(-\Delta_{b}\right)^{\frac{\alpha}{2}} u(x)=P V \int_{\mathbb{G}}(u(y)-u(x)) \tilde{R}_{-\alpha}\left(y^{-1} x\right) d y
$$

Using classical interpolation (or what is it called $\lambda$-kernel estimates in [7]) one has for $0<\alpha<Q$,

$$
\left\|R_{\alpha} u\right\|_{p} \lesssim\|u\|_{q}
$$

for $\frac{1}{p}=\frac{1}{q}-\frac{\alpha}{Q}$ and $1<q<\infty$. In the case of the Heisenberg group one in fact has more explicit computations for the operator $\mathcal{L}_{2 k}$ (see [21]). In fact, one can replace $R_{\alpha}$ by the expected Green's function of $\mathcal{L}_{2 k}$ that is

$$
G_{2 k}(x)=c_{n, k} \frac{1}{|x|^{Q-2 k}}
$$

and $\tilde{R}_{-\alpha}$ by the kernel

$$
K_{2 k}=\tilde{c}_{n, k} \frac{1}{|x|^{Q+2 k}} .
$$

The integral representation formula holds also for the operator $\mathcal{L}_{2 k}$ in our results, with $\tilde{R}_{-2 k}$ replaced by $K_{2 k}$.

\section{References}

[1] A. Ambrosetti, P. H. Rabinowitz. Dual variational methods in critical point theory and applications. J. Funct. Anal. 14 (1973), 349-381.

[2] T. Aubin. Nonlinear Analysis on Manifolds, Monge-Ampère equations. SpringerVerlag, New York, (1982).

[3] T.P. Branson, L. Fontana, C. Morpurgo. Moser-Trudinger and Beckner-Onofri's inequalities on the CR sphere. Ann. of Math. 177 (2013), 1-52.

[4] S. Chanillo. A note on commutators. Indiana Univ. Math. J. 31, no. 1, (1982), $7-16$.

[5] W.Y. Ding. On a conformally invariant elliptic equation on $\mathbb{R}^{n}$. Comm. Math. Phys. 107 (2) (1986), 331-335.

[6] F. Ferrari, B. Franchi. Harnack inequality for fractional sub-Laplacians in Carnot groups. Math. Z. 279 (1-2), (2015), 435-458. 
[7] G. Folland, E. Stein. Hardy spaces on homogeneous groups. Mathematical Notes, 28. Princeton University Press, N.J. (1982).

[8] R.L. Frank, M. del Mar González, D.D. Monticelli, J. Tan. An extension problem for the CR fractional Laplacian. Adv. Math. 270 (2015), 97-137.

[9] R. L. Frank, E. H. Lieb. Sharp constants in several inequalities on the Heisenberg group. Ann. of Math. (2) 176 (2012), no. 1, 349-381.

[10] A. Gover, C.R. Graham. CR invariant powers of the sub-Laplacian. J. Reine Angew. Math. 583 (2005), 1-27.

[11] C.R. Graham, R. Jenne, L.J. Mason, G.A.J. Sparling. Conformally invariant powers of the Laplacian I. Existence. J. Lond. Math. Soc. (2) 46(3) (1992), 557565.

[12] T. Isobe. Nonlinear Dirac equations with critical nonlinearities on compact spin manifolds. J.Funct. Anal., 260, (2011), 253-307.

[13] A. Kristály. Nodal solutions for the fractional yamabe problem on Heisenberg groups. Proc. Roy. Soc. Edinburgh Sect. A, accepted.

[14] E. Lenzmann, A. Schikorra. Sharp commutator estimates via harmonic extensions. Preprint arXiv:1609.08547.

[15] C. Liu, Y. Wang. Existence results for the fractional Q-curvature problem on three dimensional CR sphere. Preprint arXiv:1612.04015.

[16] A. Maalaoui. A Note on Commutators of the Fractional Sub-Laplacian on Carnot Groups. Preprint arXiv:1711.10298.

[17] A. Maalaoui, V. Martino Characterization of the Palais-Smale sequences for the conformal Dirac-Einstein problem and applications. submitted.

[18] A. Maalaoui, V. Martino. Changing-sign solutions for the CR-Yamabe equation. Differential Integral Equations 25 (2012), no. 7-8, 601-609.

[19] A. Maalaoui, V. Martino, G. Tralli. Complex group actions on the sphere and sign changing solutions for the CR-Yamabe equation. J. Math. Anal. Appl. 431 (2015), 126-135.

[20] R.S. Palais. The principle of symmetric criticality. Comm. Math. Phys. 69 (1) (1979), 19-30.

[21] L. Roncala, S. Thangavelub. Hardy's inequality for fractional powers of the sublaplacian on the Heisenberg group. Advances in Mathematics, 302, (2016), 106158.

[22] A. Schikorra. E-regularity for systems involving non-local, antisymmetric operators. Calc. Var. Partial Differential Equations 54, no. 4, (2015), 3531-3570. 